\title{
A Generic Three-Sided Rearrangeable Switching Network for Polygonal FPGA Design
}

\author{
Mao-Hsu Yen, ${ }^{1}$ Chu Yu, ${ }^{2}$ Horng-Ru Liao, ${ }^{3}$ and Chin-Fa Hsieh ${ }^{3}$ \\ ${ }^{1}$ Department of Computer Science and Information Engineering, National Taiwan Ocean University, 2 Pei-Ning Road, \\ Keelung 202-24, Taiwan \\ ${ }^{2}$ Department of Electronic Engineering, National Ilan University, 1, Sec. 1, Shen-Lung Road, I-Lan 260, Taiwan \\ ${ }^{3}$ Department of Electronic Engineering, China University of Science and Technology, 245, Sec. 3, Academia Road, \\ Nangang District, Taipei City 115, Taiwan \\ Correspondence should be addressed to Chu Yu; chu@niu.edu.tw
}

Received 5 August 2013; Accepted 27 September 2013

Academic Editor: Wen-Jyi Hwang

Copyright (C) 2013 Mao-Hsu Yen et al. This is an open access article distributed under the Creative Commons Attribution License, which permits unrestricted use, distribution, and reproduction in any medium, provided the original work is properly cited.

\begin{abstract}
We propose a new Polygonal Field Programmable Gate Array (PFPGA) that consists of many logic blocks interconnected by a generic three-stage three-sided rearrangeable polygonal switching network (PSN). The main component of this PSN consists of a polygonal switch block interconnected by crossbars. In comparing our PSN with a three-stage three-sided clique-based (Xilinx 4000-like FPGAs) (Palczewski; 1992) switching network of the same size and with the same number of switches, we find that the three-stage three-sided clique-based switching network is not rearrangeable. Also, the effects of the rearrangeable structure and the number of terminals on the network switch-efficiency are explored and a proper set of parameters is determined to minimize the number of switches. Moreover, we explore the effect of the PSN structure and granularity of cluster logic blocks on the switch efficiency of PFPGA. Experiments on benchmark circuits show that switches and speed performance are significantly improved. Based on experiment results, we can determine the parameters of PFPGA for the VLSI implementation.
\end{abstract}

\section{Introduction}

Field Programmable Gate Arrays (FPGA's) $[1,2]$ are now widely used for the implementation of digital circuits and many commercial products. A typical symmetric FPGA, consisting of an array of logic blocks (Ls) that can be connected by general routing resources, is shown in Figure 1. The routing resources comprise segments of wires and two kinds of blocks, switch blocks (SBs), and connection blocks (CBs). Both the switch and connection blocks contain many programmable switches. The input and output pins of a logic block are connected to its surrounding connection blocks, which in turn are connected to the switch blocks. An arbitrary digital circuit is divided into several parts such that each part is realized by a logic block; then these logic blocks are interconnected by routing resources in an FPGA.

Since the programmable switches usually have high resistance and capacitance and occupy a large area, the number of programmable switches used in an FPGA affects its speed performance, die size, and routability. The time delay along an interconnection path is significant and often exceeds the delay of logic blocks. The routing area of FPGA typically takes from 70 to $90 \%$ of the total area [3]. Intuitively, increasing the number of programmable switches of routing network in an FPGA deliver good routability. However, routing network with fewer programmable switches can reduce the impedance of interconnecting paths, and the overall interconnecting speed of the FPGA can thus be improved.

We propose a new Polygonal Field Programmable Gate Array (PFPGA) that consists of many logic blocks interconnect by a three-stage three-sided rearrangeable polygonal switching network. Figure 2 shows a model of polygonal FPGA, which consists of many clusters of logic block and $I / O$ pins interconnected by a three-stage three-sided polygonal switching network. The polygonal switching network is a three-stage three-sided switching network for connecting 


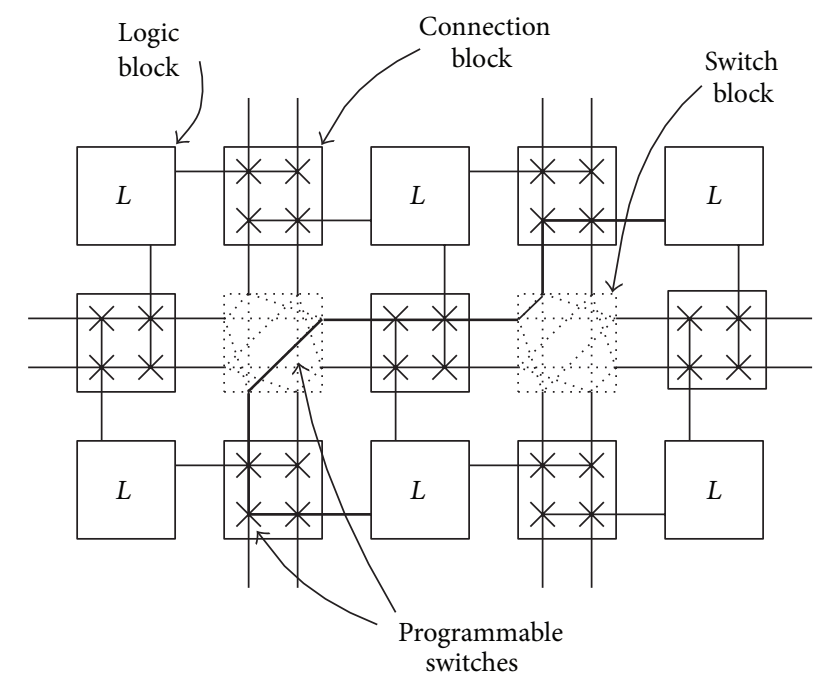

Figure 1: A typical symmetric FPGA model and its routing resources.

inputs of logic-block $\left(P_{I}\right)$, outputs of logic-block $\left(P_{\mathrm{O}}\right)$, and $I / O$ pins $\left(P_{P}\right)$ to interconnect each other. A 3-sided switch network means that we have three disjoint sets $\left(P_{P}, P_{I}\right.$, and $P_{\mathrm{O}}$ ) of terminals to be connected. Furthermore, in the 3 -stage switching network, the longest connection of terminals is the length passing through 3 switches. Through the arrangement of switches (or interconnects) in various combinations, a terminal in one set can be connected to a terminal in any other set. Assuming that all connections are point-to-point connections, the enumeration of any pair of the above three sets of terminals to be connected is called an assignment, where a terminal of the three sets can appear in at most one pair. An assignment for a network is realizable if there exist in the network disjoint paths connecting every pair of terminals given in the assignment. A 3-sided switching network is rearrangeable [4-8] if any given assignment is realizable. Since a rearrangeable switching network has better capability to accomplish a given assignment, we focus on the design of a rearrangeable 3-sided switching network for the interconnection of terminals in a PFPGA.

Studies on multistage switching networks are fruitful. Yen and Feng [5] proposed a class of $2 \log _{2} N$-stage two-sided networks, which are equivalent to Benes networks. All networks in this class are nonblocking and rearrangeable. The onestage one-sided rearrangeable switching networks have been discussed by Mitchell and Wild [6]; then, the reduction of crosspoints in the one-stage one-sided crosspoint switching network has been investigated by Varma and Chalasani [7]. Gordon and Srikanthan [8] studied another multistage onesided switching network with many $2 \times 2$ switch elements. Chang et al. and Shyu et al. [9-11] proposed universal switch blocks that can improve the routability in a Xilinx 4000like (clique-based) FPGAs routing network. Fan et al. [12] designed and proved a class of optimum Polygonal Switch $\operatorname{Block} \operatorname{PSB}(m, S)$ for all even $m$ and $S \geq 2$. Yen et al. [13] proposed rearrangeable switch network that can improve the routability for a binary fat tree. However, most of the studies are concerned with either the two-sided rearrangeable switching networks or the one-stage one-sided rearrangeable switching networks. As well as, in terms of the number of switches, the one-sided or two-sided switching network is not efficient for interconnecting the three disjoint sets of terminals. Thus, this paper presents a generic three-stage three-sided rearrangeable switching network for Polygonal Field Programmable Gate Array by using Polygonal Switching Blocks (PSBs) [14-16] and crossbars. We investigate how to use PSBs to construct a rearrangeable 3-sided Polygonal switching network (PSN) and how to minimize the number of switches repaired. We also compare our PSN with a three-stage 3-sided clique-based (Xilinx 4000-like FPGAs) polygonal switching network of the same size and with the same number of switches and derive that the three-stage 3 -sided clique-based polygonal switching network is not rearrangeable.

The next section gives a description on our polygonal FPGA model, 3-sided polygonal switching network, and some notations and definitions. In Section 3, we prove that our 3-sided switching polygonal network is rearrangeable, while Section 4 shows how we minimize the number of switches in a rearrangeable PSN. In Section 5, we propose a polygonal field programmable gate array that consists of many logic blocks interconnected by a PSN. Conclusions are reported in Section 6.

\section{Polygonal Field Programmable Gate Array}

Figure 2 shows a model of polygonal FPGA, which consists of many clusters of logic block and $I / O$ pins interconnected by a generic three-sided Polygonal Switching Network. In this study, we investigate the logic block based on a cluster of 4input look-up tables (LUTs). The reason is that most research has focused on LUT-based logic blocks, and Rose et al. [17] showed that a 4 -input LUT is the most area-efficient LUT. The three-sided polygonal switching network consists of $S$ connection blocks (CBs) and an $S$-sided polygonal switch block (PSB). Each connection block is connected to one of the $S$ sides of the polygonal switch block. All the above blocks contain many programmable switches. The polygonal switching network is a three-stage three-sided switching network for connecting inputs of logic-block, outputs of logic-block, and $I / O$ pins to interconnect each other. First, the input and output pins of cluster-based logic blocks and $I / O$ pins are connected to the related connection blocks (CBs), which are then interconnected by a polygonal switch block. That is, the first and third stages consist of $S$ CBs, and the second stage is a PSB with $S$ sides. In this PFPGA, any two terminals of the three groups in a PFPGA can be connected with fewer than three switches. Thus, the speed performance of a PFPGA can be improved.

Since routing area in an FPGA is typically more than the active area, so we investigate how to use PSBs to construct a rearrangeable 3-sided Polygonal Switching Network (PSN) and how to minimize the number of switches repaired in the following section. We also compare our PSN with a threestage 3-sided clique-based polygonal switching network of 


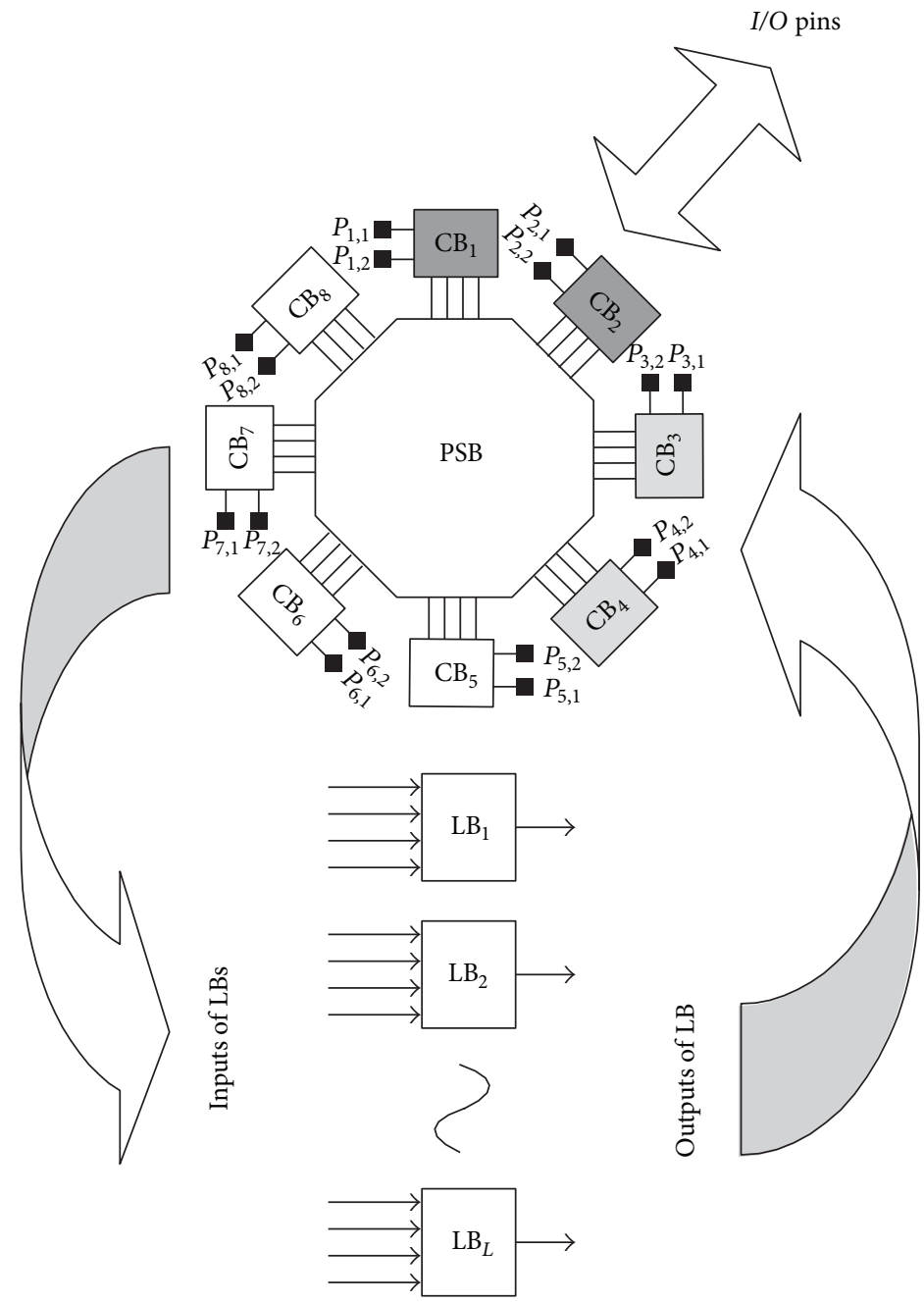

Figure 2: A polygonal FPGA model and its routing resources: $\mathrm{CB}_{P}=\left\{\mathrm{CB}_{1}, \mathrm{CB}_{2}\right\}, \mathrm{CB}_{\mathrm{O}}=\left\{\mathrm{CB}_{3}, \mathrm{CB}_{4}\right\}, \mathrm{CB}_{I}=\left\{\mathrm{CB}_{5}, \mathrm{CB}_{6}, \mathrm{CB}_{7}, \mathrm{CB}_{8}\right\}, s_{p}=2$, $s_{o}=2$, and $s_{i}=4$.

the same size and with the same number of switches and derive that the three-stage 3-sided clique-based polygonal switching network is not rearrangeable.

2.1. Three-Sided Polygonal Switching Network. The proposed generic three-stage 3-sided polygonal switching network (PSN) can be used to interconnect terminals in pairs of the three disjoint sets $\left(P_{P}, P_{\mathrm{O}}\right.$, and $\left.P_{I}\right)$. This PSN consists of $S$ crossbars (CBs) interconnected by a $S$-side polygonal switch block (PSB). Figure 3(a) shows an example of PSN, where the first and third stages are composed of $8 \mathrm{CBs}$; and the second (internal) stage is a PSB with 8 sides.

As shown in Figure 3(b), each crossbar in a PSN, denoted as $\mathrm{CB}_{i}(n, m)$ for $i=1,2, \ldots, S$, is an $n \times m$ block architecture, where $n$ is the number of external terminals connected to one of the three sets and $m$ is the number of internal terminals connected to one side of PSB, $m \in$ even [12]. We classify connection blocks $\mathrm{CB}$ s under three groups: $\mathrm{CB}_{I}$, $\mathrm{CB}_{\mathrm{O}}$, and $\mathrm{CB}_{P}$, which are used to connect the inputs and outputs of logic-blocks and $I / O$ pins, respectively. Denote that
$\mathrm{CB}_{P}=\left\{\mathrm{CB}_{1}, \mathrm{CB}_{2}, \ldots, \mathrm{CB}_{s_{p}}\right\}, \mathrm{CB}_{O}=\left\{\mathrm{CB}_{s_{p}+1}, \mathrm{CB}_{s_{p}+2}, \ldots\right.$, $\left.\mathrm{CB}_{s_{p}+s_{o}}\right\}, \mathrm{CB}_{I}=\left\{\mathrm{CB}_{s_{p}+s_{o}+1}, \mathrm{CB}_{s_{p}+s_{o}+2}, \ldots, \mathrm{CB}_{s_{p}+s_{o}+s_{i}}\right\}$, where $s_{p}, s_{o}$, and $s_{i}$ are the number of $\mathrm{CBs}$ in the $\mathrm{CB}_{P}, \mathrm{CB}_{\mathrm{O}}$, and $\mathrm{CB}_{I}$, respectively. As shown in Figure 2, the $I / O$ pins are connected to $\mathrm{CB}_{P}=\left\{\mathrm{CB}_{1}, \mathrm{CB}_{2}\right\}$, and inputs and outputs of logic-blocks are connected to $\mathrm{CB}_{I}=\left\{\mathrm{CB}_{5}, \mathrm{CB}_{6}, \mathrm{CB}_{7}, \mathrm{CB}_{8}\right\}$ and $\mathrm{CB}_{\mathrm{O}}=\left\{\mathrm{CB}_{3}, \mathrm{CB}_{4}\right\}$, respectively. Therefore, a three-sided polygonal switching network should provide $N_{I}=s_{i} \times n$, $N_{O}=s_{o} \times n$, and $N_{P}=s_{p} \times n$ external terminals to connect the inputs and outputs of logic-block and $I / O$ pins, respectively.

We label these external and internal terminals on a $\mathrm{CB}_{i}(n, m)$ as $P_{i}=\left\{p_{i, 1}, p_{i, 2}, \ldots, p_{i, n}\right\}$ and $T_{i}=\left\{t_{i, 1}, t_{i, 2}\right.$, $\left.\ldots, t_{i, m}\right\}$, respectively. We represent the external terminals of a $\mathrm{CB}_{i}(n, m) P_{P}=P_{1} \cup P_{2} \cup \cdots \cup P_{s_{p}}(I / O$ terminals $)$, $P_{\mathrm{O}}=P_{s_{p+1}} \cup P_{s_{p+2}} \cup \cdots \cup P_{s_{p}+s_{o}}$ (output terminals), and $P_{I}=$ $P_{s_{p}+s_{o}+1} \cup P_{s_{p}+s_{o}+2} \cup \cdots \cup P_{s_{p}+s_{o}+s_{i}}$ (input terminals). Similarly, the internal terminals are denoted as $T_{P}=T_{1} \cup T_{2} \cup \cdots \cup T_{s_{p}}$, $T_{O}=T_{s_{p+1}} \cup T_{s_{p+2}} \cup \cdots \cup T_{s_{p}+s_{o}}$, and $T_{I}=T_{s_{p}+s_{o}+1} \cup T_{s_{p}+s_{o}+2} \cup \ldots \cup$ $T_{s_{p}+s_{o}+s_{i}}$. In total, we have two sets of terminals: the external 


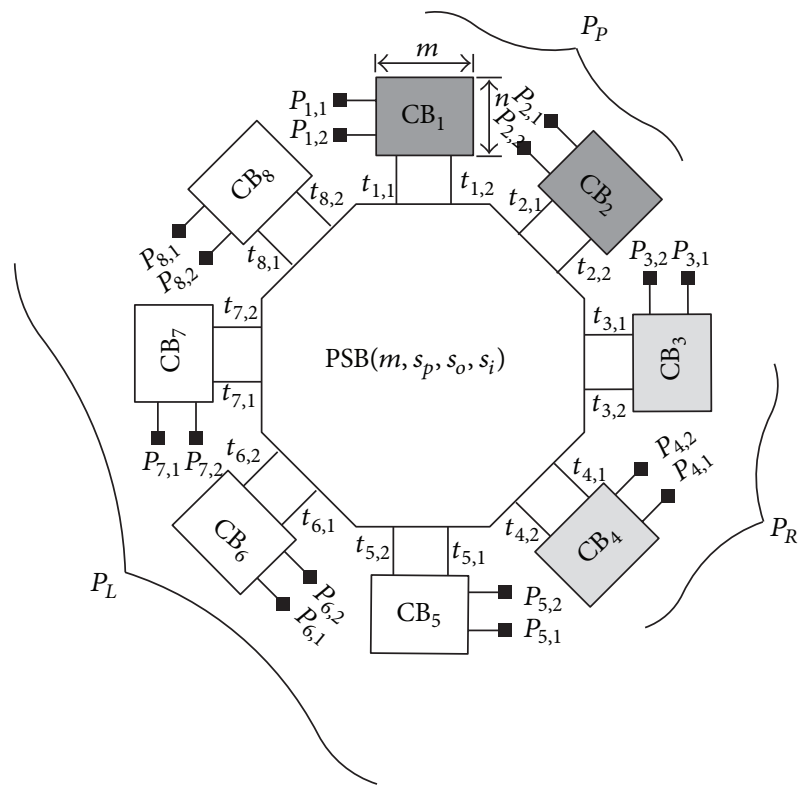

(a)

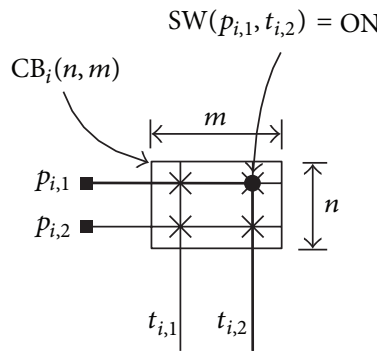

(b)

FIgURE 3: (a) A 3-sided switching network PSN $\left(n, m, s_{p}, s_{o}, s_{i}\right)$ with $n=2, m=2, s_{p}=2, s_{o}=2$, and $s_{i}=4$. (b) The connecting instance in a $\mathrm{CB}_{i}(n, m)$.

terminal set $\mathscr{P}=P_{P} \cup P_{O} \cup P_{I}$ and the internal terminal set $\mathscr{T}=T_{P} \cup T_{O} \cup T_{I}$ in a PSN. Furthermore, the set of $P_{P}, P_{O}$, and $P_{I}$ have $N_{P}=s_{p} \times n, N_{O}=s_{o} \times n$, and $N_{I}=s_{i} \times n$ external terminals, respectively. Similarly, the set of $T_{P}, T_{O}$, and $T_{I}$ have $M_{P}=s_{p} \times m, M_{O}=s_{o} \times m$, and $M_{I}=s_{i} \times m$ internal terminals on $S \mathrm{CB}(n, m)$ crossbars. For example, Figure 3(a) shows a 3-sided switching network PSN with $P_{P}=P_{1} \cup P_{2}$, $P_{O}=P_{3} \cup P_{4}$, and $P_{I}=P_{5} \cup P_{6} \cup P_{7} \cup P_{8}$, where $P_{i}=\left\{p_{i, 1}, p_{i, 2}\right\}$, $i=1,2, \ldots, 8$; and with $T_{P}=T_{1} \cup T_{2}, T_{O}=T_{3} \cup T_{4}$, and $T_{I}=T_{5} \cup T_{6} \cup T_{7} \cup T_{8}$, where $T_{i}=\left\{t_{i, 1}, t_{i, 2}\right\}, i=1,2, \ldots, 8$. Any pair of external terminals from two sets of $P_{P}, P_{O}$, and $P_{I}$ can be connected to each other by using a 3-sided switching network PSN, but no pair external terminals from the same set can be interconnected.

Through the $n \times m$ programmable and electrically noninteracting switches in a crossbar $\mathrm{CB}_{i}(n, m)$, every external terminal in $P_{i}$ can be connected to a free internal terminal in $T_{i}$ without any blocking. For example, if switch $\mathrm{SW}\left(p_{i, j}, t_{i, h}\right)$ is programmed to be "ON", then connection $\left(p_{i, j}, t_{i, h}\right)$ between an external terminal $p_{i, j}$ and an internal terminal $t_{i, h}$ is established, where $1 \leq i \leq S, 1 \leq j \leq n$, and $1 \leq h \leq m$. Figure 3(b) shows a routing example, where $p_{i, 1}$ is connected to $t_{i, 2}$ by programming $S W\left(p_{i, 1}, t_{i, 2}\right)$, and this routing solution is illustrated by thick lines. In such a way, an external terminal can be connected to an internal terminal by programming one switch in $\mathrm{CB}_{i}(n, m)$.

The polygonal switch block in a PSN, denoted as a $\operatorname{PSB}\left(m, s_{p}, s_{o}, s_{i}\right)$, is an $S$-side switch block with $m$ internal terminals on each side of the block, as shown in Figures 4(a) and 5(a). Label the terminals on the $i$ th side of a $\operatorname{PSB}\left(m, s_{p}, s_{o}, s_{i}\right)$ as $T_{i}=\left\{t_{i, 1}, t_{i, 2}, \ldots, t_{i, m}\right\}$ for $1 \leq i \leq S$. Remember that internal terminals $\mathscr{T}=T_{P} \cup T_{O} \cup T_{I}$, where $T_{P}=T_{1} \cup T_{2} \cup \cdots \cup T_{s_{p}}, T_{O}=T_{s_{p}+1} \cup T_{s_{p}+2} \cup \cdots \cup T_{s_{p}+s_{o}}$, and $T_{I}=T_{s_{p}+s_{o}+1} \cup T_{s_{p}+s_{o}+2} \cup \cdots \cup T_{s_{p}+s_{o}+s_{i}}$. If a switch $\operatorname{SW}\left(t_{i, j}, t_{k, l}\right)$ exists in $\operatorname{PSB}\left(m, s_{p}, s_{o}, s_{i}\right)$ and is programmed to be "ON", then a connection between terminals $t_{i, j}$ and $t_{k, l}$ is established, where $t_{i, j}$ and $t_{k, l}$ belong to two different sets of $T_{P}, T_{O}$, and $T_{I}$. To form a 3-sided switching network PSN we need $S \mathrm{CB}(n, m)$ crossbars connected to a polygonal switch block $\operatorname{PSB}\left(m, s_{p}, s_{o}, s_{i}\right)$. Thus, a 3-sided switching network can be completely characterized by five parameters: $n, m, s_{p}, s_{o}$, and $s_{i}$, and denoted as $\operatorname{PSN}\left(n, m, s_{p}, s_{o}, s_{i}\right)$, for $n \geq 2, m \in$ even, $s_{p} \geq 1, s_{o} \geq 1, s_{i} \geq 1$, and $S=s_{p}+s_{o}+s_{i}$. For example, Figure 3(a) shows a PSN with $n=2, m=2, s_{p}=2, s_{o}=2$, and $s_{i}=4$.

As shown in Figures 4(a) and 5(a), we will study the 3-sided switching network with two different topologies of polygonal switch blocks in this paper, denoted as $\operatorname{PSB}_{C}\left(m, s_{p}, s_{o}, s_{i}\right)$ and $\operatorname{PSB}_{\mathrm{SU}}\left(m, s_{p}, s_{o}, s_{i}\right)$, respectively. Algorithm 1 constructs a clique-based polygonal switch block $\operatorname{PSB}_{C}\left(m, s_{p}, s_{o}, s_{i}\right)$. As shown in Figure $4, \operatorname{aPSB}_{C}\left(m, s_{p}, s_{o}, s_{i}\right)$ is equivalent to $m$ isolated $\operatorname{PSB}_{C}\left(1, s_{p}, s_{o}, s_{i}\right)$. In Figure 6 we show a 3 -sided switching network, $\operatorname{PSN}_{C}\left(n, m, s_{p}, s_{o}, s_{i}\right)$ constructed with a $\operatorname{PSB}_{C}\left(m, s_{p}, s_{o}, s_{i}\right)$. Algorithm 2 constructs the polygonal switch block $\mathrm{PSB}_{\mathrm{SU}}\left(m, s_{p}, s_{o}, s_{i}\right)$. As shown in Figure 5, a $\operatorname{PSB}_{\mathrm{SU}}\left(m, s_{p}, s_{o}, s_{i}\right)$ is equivalent to $m / 2$ isolated $\operatorname{PSB}_{\mathrm{SU}}\left(2, s_{p}, s_{o}, s_{i}\right), m \in$ even. A 3 -sided switching network $\operatorname{PSN}_{\mathrm{SU}}\left(n, m, s_{p}, s_{o}, s_{i}\right)$ consisting of $S \mathrm{CB}(n, m)$ crossbars connected to a $\operatorname{PSB}_{\mathrm{SU}}\left(m, s_{p}, s_{o}, s_{i}\right)$ is shown in Figure 7. Table 1 lists some symbols of 3-sided switching network specified in this paper.

Note that we do not allow any two external terminals from the same set of $P_{P}, P_{O}$, and $P_{I}$ to be interconnected in a $\operatorname{PSN}\left(n, m, s_{p}, s_{o}, s_{i}\right)$. Similarly, we do not allow any two internal terminals from the same set of $T_{P}, T_{O}$, and $T_{I}$ to be interconnected in a $\operatorname{PSB}\left(m, s_{p}, s_{o}, s_{i}\right)$. 


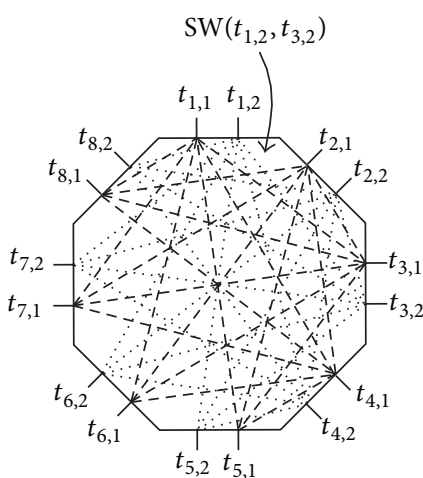

(a)

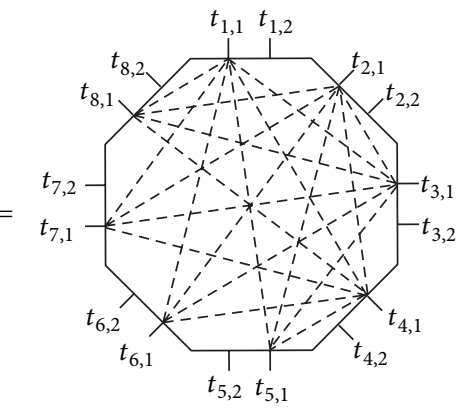

(b)

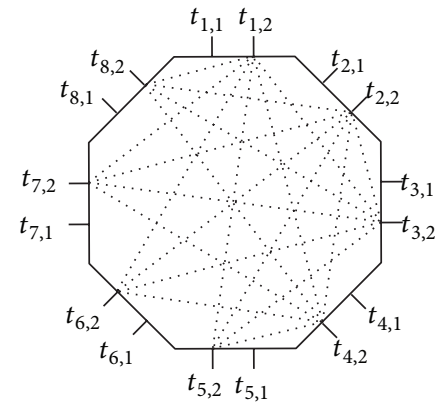

Figure 4: (a) A polygonal switch block $\operatorname{PSB}_{C}\left(m, s_{p}, s_{o}, s_{i}\right)$ with $m=2, s_{p}=2, s_{o}=2$, and $s_{i}=4$; and (b) Its correspondence to two $\operatorname{PSB}_{C}(1,2,2,4)$.

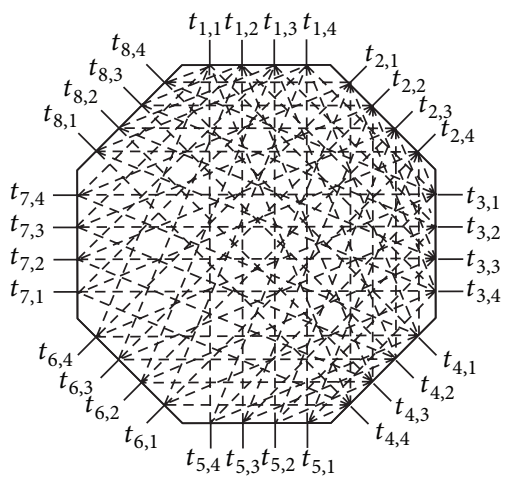

(a)

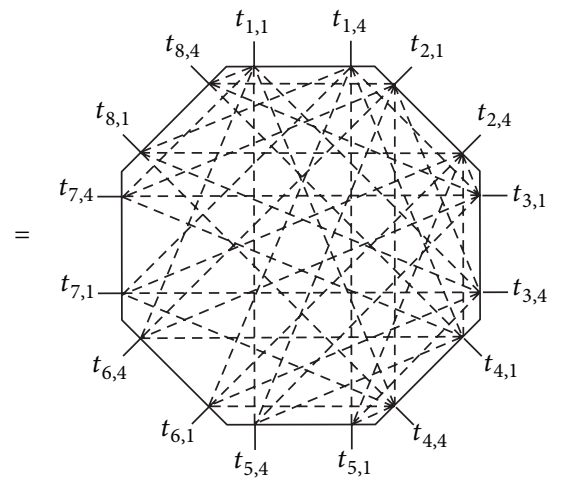

(b)

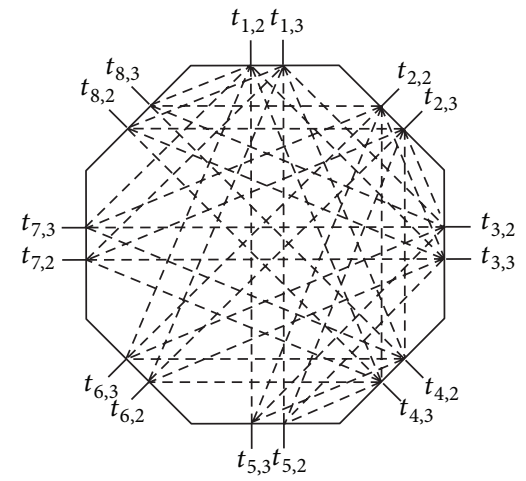

FIGURE 5: A polygonal switch block $\operatorname{PSB}_{\mathrm{SU}}\left(m, s_{p}, s_{o}, s_{i}\right)$ with $m=4, s_{p}=2, s_{o}=2$, and $s_{i}=4$. (b) Its correspondence to two PSB $\mathrm{SU}(2,2,2,4)$.

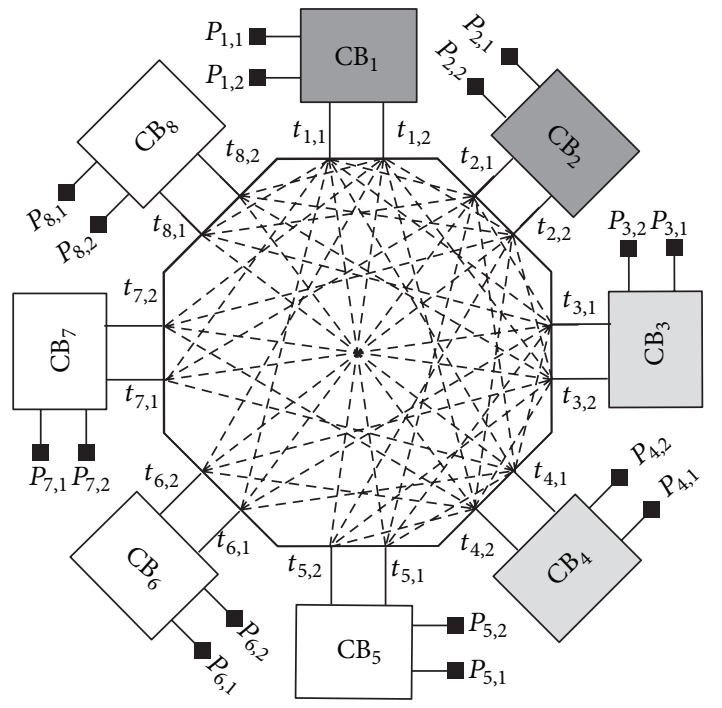

FIGURE 6: $\operatorname{APSN}_{C}\left(n, m, s_{p}, s_{o}, s_{i}\right)$ with $n=2, m=2, s_{p}=2, s_{o}=2$, and $s_{i}=4$.

For example, Figures 4(a) and 5(a) show that $\operatorname{PSB}_{C}(2,2,2,4)$ and $\operatorname{PSB}_{\mathrm{SU}}(4,2,2,4)$ do not have any switches between the two sets of $T_{1}$ and $T_{2}$ from the same $T_{P}$ for the interconnection. That is, in either a $\operatorname{PSN}_{C}(2,2,2,2,4)$ (Figure 6)

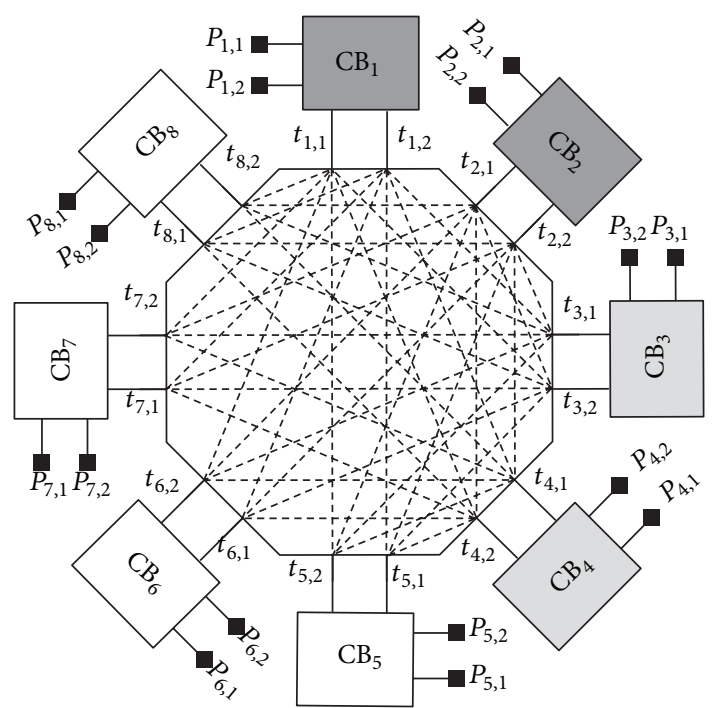

FigUre 7: $\operatorname{A~PSN~}_{\mathrm{SU}}\left(n, m, s_{p}, s_{o}, s_{i}\right)$ with $n=2, m=2, s_{p}=2, s_{o}=2$, and $s_{i}=4$.

or a $\operatorname{PSN}_{\mathrm{SU}}(2,2,2,2,4)$ (Figure 7 ) no interconnections of external terminals from the same sets of $P_{1}$ and $P_{2}$ through $\operatorname{PSB}_{C}(2,2,2,4)$ or $\operatorname{PSB}_{\mathrm{SU}}(2,2,2,4)$ are allowed. In a $\operatorname{PSB}_{\mathrm{SU}}\left(m, s_{p}, s_{o}, s_{i}\right)$ or $\operatorname{PSB}_{C}\left(m, s_{p}, s_{o}, s_{i}\right)$, since a terminal 
TABLE 1: Summary of symbols.

\begin{tabular}{|c|c|}
\hline Symbol & Description \\
\hline $\mathrm{CB}(n, m)$ & $n \times m$ crossbar \\
\hline $\operatorname{PSB}\left(m, s_{p}, s_{o}, s_{i}\right)$ & $S$-side PSB with $m$ internal terminals, $S=s_{p}+s_{o}+s_{i}$ \\
\hline $\operatorname{PSB}_{C}\left(m, s_{p}, s_{o}, s_{i}\right)$ & A clique-based $\operatorname{PSB}\left(m, s_{p}, s_{o}, s_{i}\right)$ \\
\hline $\operatorname{PSB}_{U}\left(m, s_{p}, s_{o}, s_{i}\right)$ & A universal $\operatorname{PSB}\left(m, s_{p}, s_{o}, s_{i}\right)$ \\
\hline $\operatorname{PSB}_{\mathrm{SU}}\left(m, s_{p}, s_{o}, s_{i}\right)$ & A semiuniversal $\operatorname{PSB}\left(m, s_{p}, s_{o}, s_{i}\right)$ \\
\hline $\operatorname{PSN}\left(n, m, s_{p}, s_{o}, s_{i}\right)$ & $S \mathrm{CB}(n, m))^{\prime} \mathrm{s}+\operatorname{PSB}\left(m, s_{p}, s_{o}, s_{i}\right)$ \\
\hline $\operatorname{PSN}_{C}\left(n, m, s_{p}, s_{o}, s_{i}\right)$ & $\operatorname{A} \operatorname{PSN}\left(n, m, s_{p}, s_{o}, s_{i}\right)$ with $\operatorname{PSB}_{C}$ \\
\hline $\operatorname{PSN}_{U}\left(n, m, s_{p}, s_{o}, s_{i}\right)$ & $\operatorname{A} \operatorname{PSN}\left(n, m, s_{p}, s_{o}, s_{i}\right)$ with $\operatorname{PSB}_{U}$ \\
\hline $\operatorname{PSN}_{\mathrm{SU}}\left(n, m, s_{p}, s_{o}, s_{i}\right)$ & $\operatorname{A} \operatorname{PSN}\left(n, m, s_{p}, s_{o}, s_{i}\right)$ with $\operatorname{PSB}_{\mathrm{SU}}$ \\
\hline $\mathrm{SW}_{\mathrm{SU}}\left(n, m, s_{p}, s_{o}, s_{i}\right)$ & The number of switches in a $\operatorname{PSN}_{\mathrm{SU}}\left(n, m, s_{p}, s_{o}, s_{i}\right)$ \\
\hline
\end{tabular}

from a side in one set of $T_{P}, T_{O}$, and $T_{I}$ should be connected to any other terminal from the sides in the remaining two sets through switches, a polygonal switch block needs at least $m\left(s_{p} s_{o}+s_{p} s_{i}+s_{o} s_{i}\right)$ switches. Assume that both 3-sided switching networks $\operatorname{PSN}_{\mathrm{SU}}\left(n, m, s_{p}, s_{o}, s_{i}\right)$ and $\operatorname{PSN}_{C}\left(n, m, s_{p}, s_{o}, s_{i}\right)$ have the same size and the same number of switches. We will show that the three-stage 3-sided switching network $\operatorname{PSN}_{\mathrm{SU}}\left(n, m, s_{p}, s_{o}, s_{i}\right)$ with $m \geq n$ is rearrangeable and $m$ is even [12], but the $\operatorname{PSN}_{C}\left(n, m, s_{p}, s_{o}, s_{i}\right)$ with $m=n$ is not rearrangeable.

In a $\operatorname{PSN}\left(n, m, s_{p}, s_{o}, s_{i}\right)$, the connection pair $\left(p_{i, j}, p_{k, l}\right)$ is a point-to-point connection, where $p_{i, j}$ and $p_{k, l}$ belong to different sets of $P_{P}, P_{O}$, and $P_{I}$. An assignment $\mathrm{AS}=$ $\left\{\left(p_{i, j}, p_{k, l}\right)\right\}$ represents a set of connection pairs to be connected; thus each external terminal appears in at most one pair. A PSN $\left(n, m, s_{p}, s_{o}, s_{i}\right)$ switching network is rearrangeable if any assignment AS is realizable (routable).

Note that a connection pair $\left(p_{i, j}, p_{k, l}\right)$ belonging to $\mathrm{AS}$ is to be connected by passing through blocks $\mathrm{CB}-$ $\mathrm{PSB}-\mathrm{CB}$. Given an AS on a $\operatorname{PSB}\left(m, s_{p}, s_{o}, s_{i}\right)$, each pair of connection is accomplished using terminals from two different sides of $T_{P}, T_{O}$, and $T_{I}$; we can thus classify these connections passing through a $\operatorname{PSB}\left(m, s_{p}, s_{o}, s_{i}\right)$ into $\left(s_{p} s_{o}+\right.$ $\left.s_{p} s_{o}+s_{o} s_{i}\right)$ types of connections. Figure 8 shows the twenty possible types of connections in an eight-side switch block $\operatorname{PSB}(m, 2,2,4)$. A routing requirement vector $(\mathrm{RRV}) \vec{r}$ [9-11] for a $\operatorname{PSB}\left(m, s_{p}, s_{o}, s_{i}\right)$ is a $\left(s_{p} s_{o}+s_{p} s_{o}+s_{o} s_{i}\right)$-tuple $\left(r_{1, s_{p}+1}\right.$, $r_{1, s_{p}+2}, \ldots, r_{1, s_{p}+s_{o}+s_{i}}, r_{2, s_{p}+1}, \ldots, r_{2, s_{p}+s_{o}+s_{i}}, \ldots, r_{s_{p}, s_{p}+1}, \ldots$, $\left.r_{s_{p}, s_{p}+s_{o}+s_{i}}, \ldots, r_{s_{p}+s_{o}, s_{p}+s_{o}+s_{i}}\right)$, where $r_{i, k}$ represents the number of type- $(i, k)$ connections between $T_{i}$ and $T_{k}$ required to be connected through a $\operatorname{PSB}\left(m, s_{p}, s_{o}, s_{i}\right) ; 0 \leq r_{i, k} \leq m$ for $1 \leq$ $i<k \leq S$. In other words, $r_{i, k}$ is the number of connections $\left\{\left(p_{i, j}, p_{k, l}\right)\right\}$ in AS to be connected through the two sides $T_{i}$ and $T_{k}$ on $\operatorname{PSB}\left(m, s_{p}, s_{o}, s_{i}\right), 1 \leq j, l \leq m$, where $T_{i}$ and $T_{k}$ belong to any two different sets of $T_{P}, T_{O}$, and $T_{I}$. An RRV $\vec{r}$ is said to be realizable (routable) on a $\operatorname{PSB}\left(m, s_{p}, s_{o}, s_{i}\right)$ if there exist disjoint paths for $\vec{r}$ on a $\operatorname{PSB}\left(m, s_{p}, s_{o}, s_{i}\right)$. Figure 8 shows an RRV $\vec{r}=\left(r_{1,3}, r_{1,4}, r_{1,5}, r_{1,6}, r_{1,7}, r_{1,8}, r_{2,3}, r_{2,4}, r_{2,5}, r_{2,6}, r_{2,7}\right.$, $\left.r_{2,8}, r_{3,5}, r_{3,6}, r_{3,7}, r_{3,8}, r_{4,5}, r_{4,6}, r_{4,7}, r_{4,8}\right)$ for PSB with eight sides.

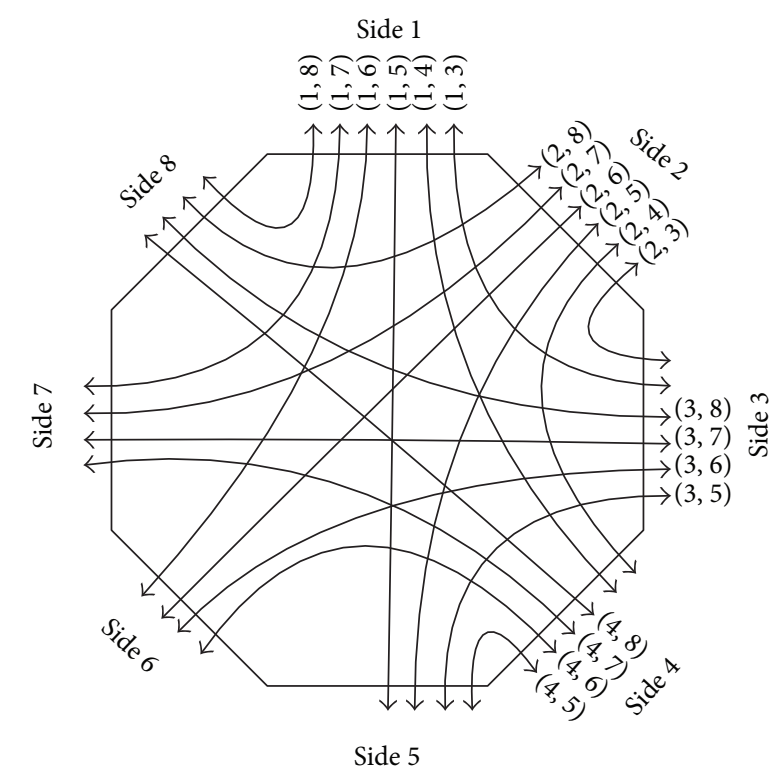

FIGURE 8: Twenty possible types of connections in an eight-side switch block.

For example, Figure 9(a) shows a routing instance with three nets corresponding to the RRV $\vec{r}=\left(1_{1,3}, 0,0,0,1_{1,7}\right.$, $\left.0,0,0,0,0,0,0,0,0,1_{3,7}, 0,0,0,0,0\right)$, and we try to route this RRV using the two different polygonal switch blocks $\operatorname{PSB}_{C}(2,2,2,4)$ and $\operatorname{PSB}_{\mathrm{SU}}(2,2,2,4)$ from Figures $4(\mathrm{a})$ and 5(b), respectively. As shown in Figures 9(b) and 9(c), however, there is always one net that cannot be routed on a $\operatorname{PSB}_{C}(2,2,2,4)$. Thus, this RRV $\left(1_{1,3}, 0,0,0,1_{1,7}, 0,0,0,0,0\right.$, $\left.0,0,0,0,1_{3,7}, 0,0,0,0,0\right)$ is not routable on a $\operatorname{PSB}_{C}(2,2,2,4)$. Instances of the same RRV $\vec{r}$ routable on a $\operatorname{PSB}_{\mathrm{SU}}(2,2,2,4)$ are shown in Figures 10(a) and 10(b), where the routing solutions are illustrated by thick lines.

Each connection pair $\left(p_{i, j}, p_{k, l}\right) \in \mathrm{AS}$ is required to be connected by passing through $\mathrm{CB}_{i}-\mathrm{PSB}-\mathrm{CB}_{k}$ in $\operatorname{arN}\left(n, m, s_{p}, s_{o}, s_{i}\right)$. Given an $\mathrm{AS}=\left\{\left(p_{i, j}, p_{k, l}\right)\right\}$, we want to obtain an RRV $\vec{r}=\left(r_{1, s_{p}+1}, r_{1, s_{p}+2}, \ldots\right.$, $r_{1, s_{p}+s_{o}+s_{i}}, r_{2, s_{p}+1}, \ldots, r_{2, s_{p}+s_{o}+s_{i}}, \ldots, r_{s_{p}, s_{p}+1}, \ldots, r_{s_{p}, s_{p}+s_{o}+s_{i}}, \ldots$, $\left.r_{s_{p}+s_{o}, s_{p}+s_{o}+s_{i}}\right)$ to be realized on a $\operatorname{PSB}\left(m, s_{p}, s_{o}, s_{i}\right)$, where $r_{i, k}$ is the number of the connection pairs $\left(p_{i, j}, p_{k, l}\right)$ required 


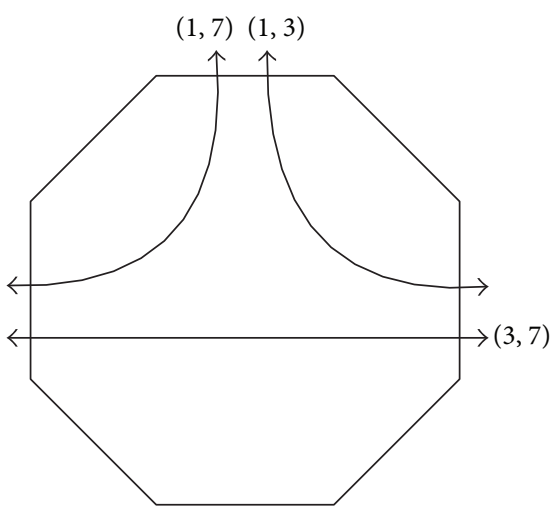

(a)

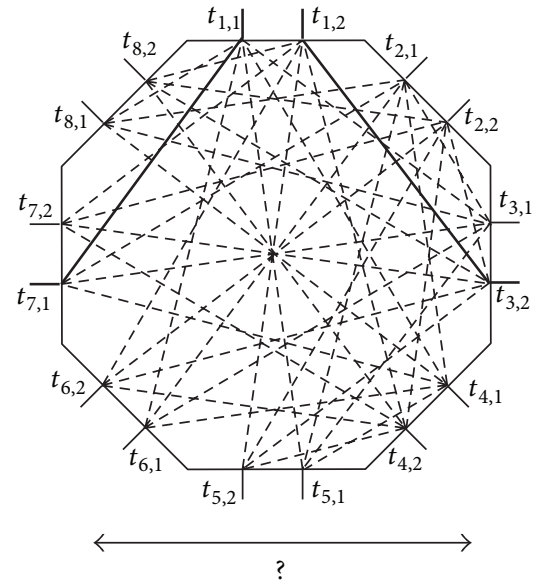

(b)

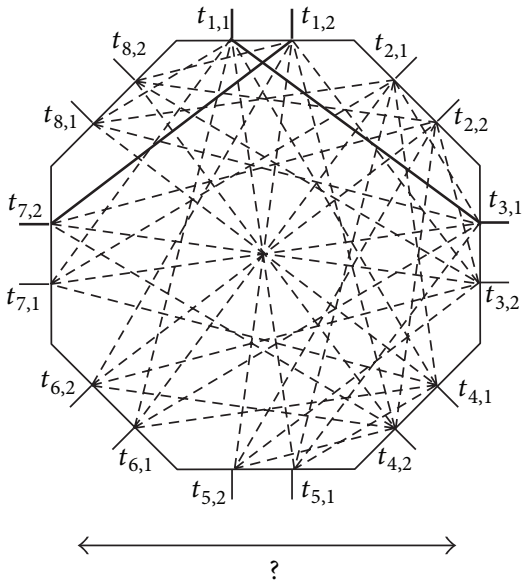

(c)

FIgURE 9: Examples of routing on $\operatorname{PSB}_{C}(2,2,2,4)$. (a) An RRV instance $\left(1_{1,3}, 0,0,0,1_{1,7}, 0,0,0,0,0,0,0,0,0,1_{3,7}, 0,0,0,0,0\right)$. (b), (c) The $\mathrm{RRV}$ is not routable on a $\operatorname{PSB}_{C}(2,2,2,4)$.

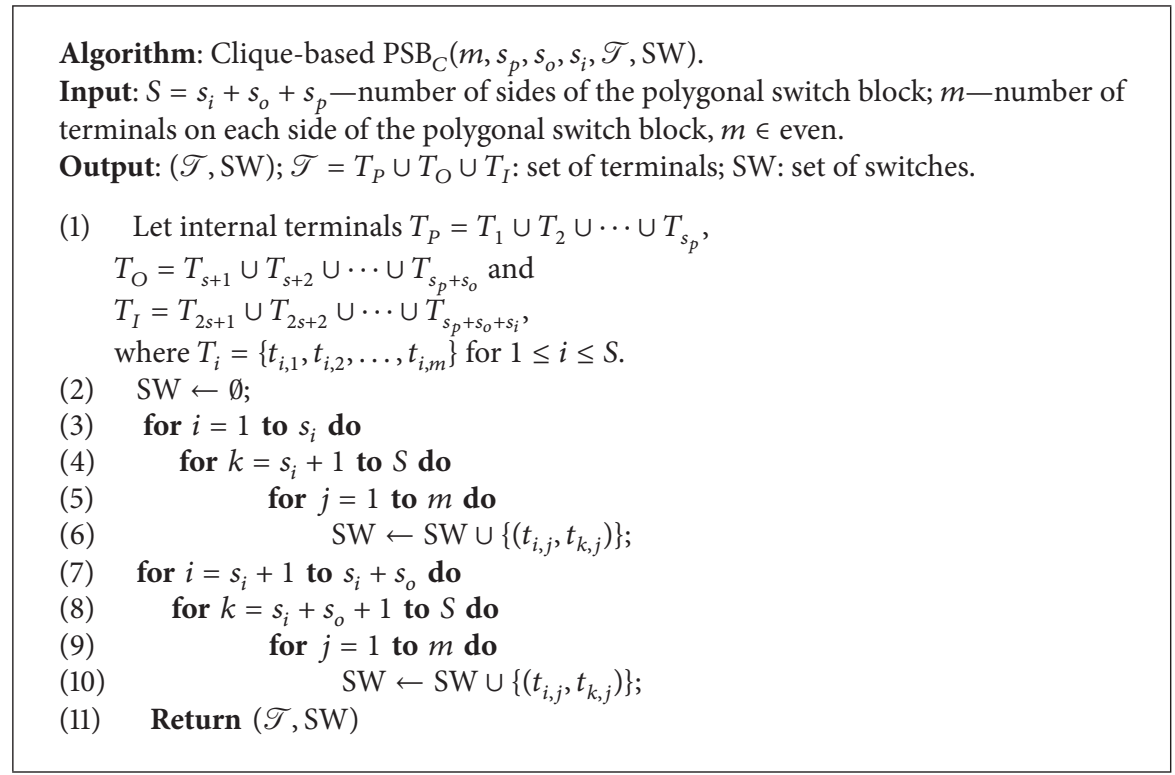

Algorithm 1: Algorithm for constructing an $S$-sided clique-based switch block of size $m$.

to be connected through $\operatorname{PSB}\left(m, s_{p}, s_{o}, s_{i}\right)$. For example, let $\mathrm{AS}=\left\{\left(p_{1,1}, p_{7,2}\right),\left(p_{1,2}, p_{3,1}\right),\left(p_{3,2}, p_{7,1}\right)\right\}$ to be routed on a

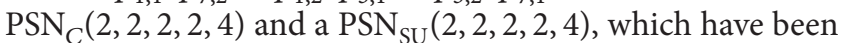
respectively shown in Figures 6 and 7. The RRV for the given AS on $\operatorname{PSB}(2,2,2,4)$ is $\vec{r}=\left(1_{1,3}, 0,0,0,1_{1,7}, 0,0,0,0,0,0\right.$, $\left.0,0,0,1_{3,7}, 0,0,0,0,0\right)$. We have already shown in Figures 10 (a) or 10(b) that the RRV $\vec{r}=\left(1_{1,3}, 0,0,0,1_{1,7}, 0,0,0,0,0,0\right.$, $\left.0,0,0,1_{3,7}, 0,0,0,0,0\right)$ can be routed on a $\operatorname{PSB}_{\mathrm{SU}}(2,2,2,4)$. Since any external terminal in $P_{i}$ can be connected to a free internal terminal in $T_{i}$ without blocking through a crossbar $\mathrm{CB}_{i}(n, m)$, we show in Figures 11(a) and 11(b) two possible routing solutions for the given AS on a $\operatorname{PSN}_{\mathrm{SU}}(2,2,2,2,4)$ switching network. Instances in Figures 12(a) and 12(b) show that the same AS is not routable on a $\operatorname{PSN}_{C}(2,2,2,2,4)$ switching network, because the RRV $\vec{r}=\left(1_{1,3}, 0,0,0,1_{1,7}\right.$, $\left.0,0,0,0,0,0,0,0,0,1_{3,7}, 0,0,0,0,0\right)$ contains at least one connection that cannot be routed on a $\operatorname{PSB}_{C}(2,2,2,4)$ as already shown in Figures 9(b) and 9(c). Therefore, we conclude a $\operatorname{PSN}_{C}\left(n, m, s_{p}, s_{o}, s_{i}\right)$ switch network constructed with a $\operatorname{PSB}_{C}(m, s)$, where $m=n$ is not rearrangeable. In the following, we prove that a $\operatorname{PSN}_{C}\left(n, m, s_{p}, s_{o}, s_{i}\right)$ with $m \leq n$ is not rearrangeable.

Theorem 1. A 3-sided switching network $\operatorname{PSN}_{C}\left(n, m, s_{p}, s_{o}, s_{i}\right)$ is not rearrangeable for $m \leq n, m \in$ even, $S=s_{p}+s_{o}+s_{i}$, $s_{p} \geq 1, s_{o} \geq 1$, and $s_{i} \geq 1$. 
Algorithm: Semi-universal $\operatorname{PSB}_{\mathrm{SU}}\left(m, s_{p}, s_{o}, s_{i}, \mathscr{T}, \mathrm{SW}\right)$.

Input: $S=s_{i}+s_{o}+s_{p}$-number of sides of the polygonal switch block; $m$-number of terminals on each side of the polygonal switch block, $m \in$ even.

Output: $(\mathscr{T}, \mathrm{SW}) ; \mathscr{T}=T_{P} \cup T_{O} \cup T_{I}$ : set of terminals; SW: set of switches.

(1) Let internal terminals $T_{P}=T_{1} \cup T_{2} \cup \cdots \cup T_{s_{p}}$,

$T_{O}=T_{s_{p}+1} \cup T_{s_{p}+2} \cup \cdots \cup T_{s_{p}+s_{o}}$ and

$T_{I}=T_{s_{p}+s_{0}+1} \cup T_{s_{p}+s_{o}+2} \cup \cdots \cup T_{s_{p}+s_{o}+s_{i}}$,

where $T_{i}=\left\{t_{i, 1}, t_{i, 2}, \ldots, t_{i, m}\right\}$ for $1 \leq i \leq S$.

(2) $\mathrm{SW} \leftarrow \emptyset$;

(3) for $i=1$ to $s_{p}$ do

(4) for $k=s_{p}+1$ to $S$ do

(5) for $j=1$ to $m$ do

(6) $\quad \mathrm{SW} \leftarrow \mathrm{SW} \cup\left\{\left(t_{i, j}, t_{k, m-j+1}\right)\right\}$;

(7) for $i=s_{p}+1$ to $s_{p}+s_{o}$ do

(8) for $k=s_{p}+s_{o}+1$ to $s_{p}+s_{o}+s_{i}$ do

(9) for $j=1$ to $m$ do

(10) $\quad \mathrm{SW} \leftarrow \mathrm{SW} \cup\left\{\left(t_{i, j}, t_{k, m-j+1}\right)\right\}$;

(11) $\quad$ Return $(\mathscr{T}, \mathrm{SW})$

Algorithm 2: Algorithm for constructing an $S$-sided semi-universal switch block of size $m$.

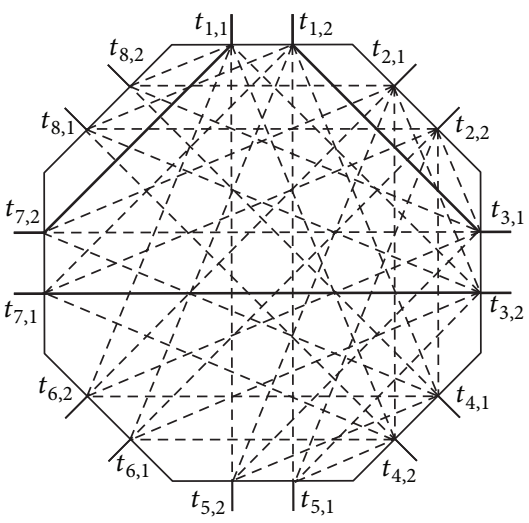

(a)

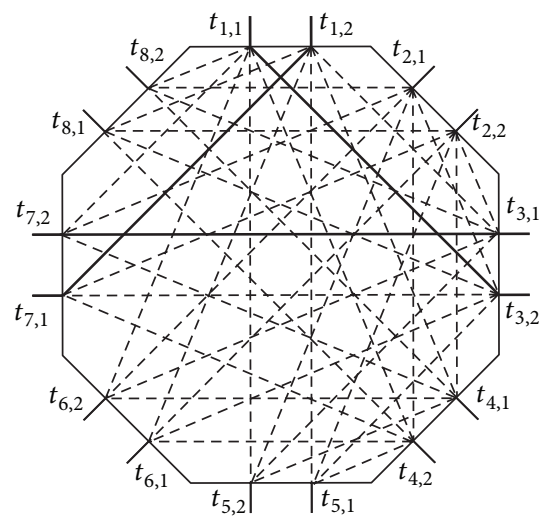

(b)

Figure 10: (a), (b) An RRV $\left(1_{1,3}, 0,0,0,1_{1,7}, 0,0,0,0,0,0,0,0,0,1_{3,7}, 0,0,0,0,0\right)$ routable on a $\operatorname{PSB}_{\mathrm{SU}}(2,2,2,4)$.

Proof. Observably, if a $\operatorname{PSN}_{C}\left(n, m, s_{p}, s_{o}, s_{i}\right)$ with $m=n$ is not rearrangeable, then a $\operatorname{PSN}_{C}\left(n, m, s_{p}, s_{o}, s_{i}\right)$ with $m<$ $n$ is not rearrangeable. Thus, we need to prove that a $\operatorname{PSN}_{C}\left(n, n, s_{p}, s_{o}, s_{i}\right)$ is not rearrangeable.

Arbitrarily select three sides $i, k$, and $u$ of a $\operatorname{PSN}_{C}\left(n, n, s_{p}, s_{o}, s_{i}\right), 1 \leq i \leq s_{p}<k \leq s_{p}+s_{o}<u \leq S$, we form an assignment AS $=\left\{\left(p_{i, 1}, p_{u, 1}\right),\left(p_{i, 2}, p_{u, 2}\right), \ldots\right.$, $\left.\left(p_{i, n-1}, p_{u, n-1}\right), \quad\left(p_{i, n}, p_{k, n}\right), \quad\left(p_{k, 1}, p_{u, n}\right)\right\}$ to be connected between the $P_{i} \in P_{P}, P_{k} \in P_{\mathrm{O}}$, and $P_{u} \in P_{I}$ on a $\operatorname{PSN}_{C}\left(n, n, s_{p}, s_{o}, s_{i}\right)$. The RRV for routing $\operatorname{AS}_{D}$ on a $\mathrm{PSB}_{C}\left(n, s_{p}, s_{o}, s_{i}\right)$ is $\vec{r}=\left(0, \ldots, 0, r_{i, k}=1,0, \ldots, 0, r_{i, u}=\right.$ $\left.n-1,0, \ldots, 0, r_{k, u}=1,0, \ldots, 0\right)$, as shown in Figure 4(a). Since a $\operatorname{PSB}_{C}\left(n, s_{p}, s_{o}, s_{i}\right)$ is equivalent to $n$ isolated $\operatorname{PSB}_{C}\left(1, s_{p}, s_{o}, s_{i}\right)$ 's, the first $r_{i, u}=(n-1)$ can be realizable on $(n-1)$ isolated $\operatorname{PSB}_{C}\left(1, s_{p}, s_{o}, s_{i}\right)$ 's, as shown in Figure 4(b). But, we cannot find enough disjoint paths to simultaneously realize an $r_{i, k}=1$ and an $r_{k, u}=1$ on the last $\operatorname{PSB}_{C}\left(1, s_{p}, s_{o}, s_{i}\right)$, as shown in Figure 4(b). Thus, this AS cannot be realizable on a $\operatorname{PSN}_{C}\left(n, n, s_{p}, s_{o}, s_{i}\right)$, because this RRV $\vec{r}$ contains at least one connection that cannot be routed on a $\operatorname{PSB}_{C}\left(n, s_{p}, s_{o}, s_{i}\right)$. Therefore, the $\operatorname{PSN}_{C}\left(n, n, s_{p}, s_{o}, s_{i}\right)$ is not rearrangeable.

In the following, we will explore the properties of the semiuniversal and polygonal switch block $\operatorname{PSB}_{\mathrm{SU}}\left(m, s_{p}, s_{o}, s_{i}\right)$ and show a $\operatorname{PSN}_{\mathrm{SU}}\left(n, m, s_{p}, s_{o}, s_{i}\right)$ switching network constructed with semiuniversal $\operatorname{PSB}_{\mathrm{SU}}\left(m, s_{p}, s_{o}, s_{i}\right)$, where $m \geq n$ is rearrangeable.

2.2. Semiuniversal and Polygonal Switch Blocks. Chang et al. and Shyu et al. [9-11] proposed a universal polygonal switch block $\operatorname{PSB}_{U}(m, S)$ to improve the routability in a Field Programmable Gate Array routing network. Fan et al. [12] designed and proved a class of optimum $\operatorname{PSB}(m, S)$ for all even $m$ and $S \geq 2$. $\operatorname{APSB}_{U}(m, S)$ is an $S$-sided switch block with $m$ terminals on each side of the block; and a terminal from one 


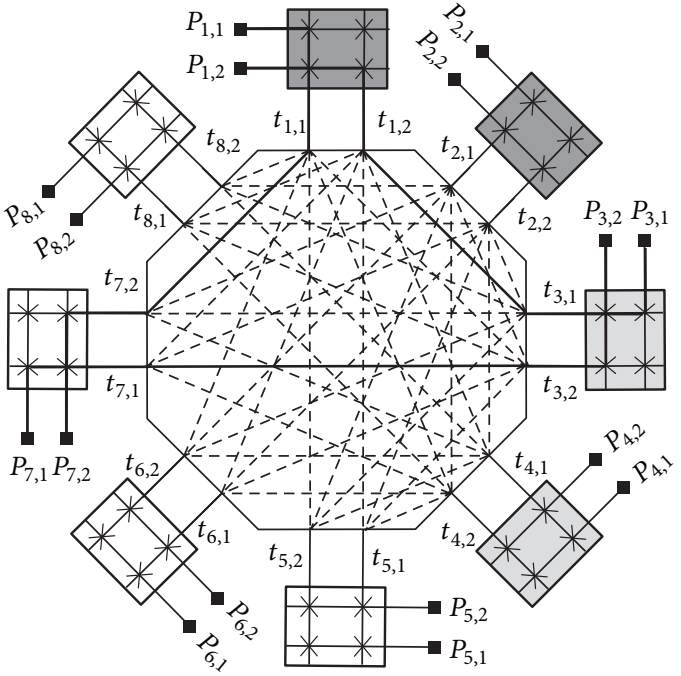

(a)

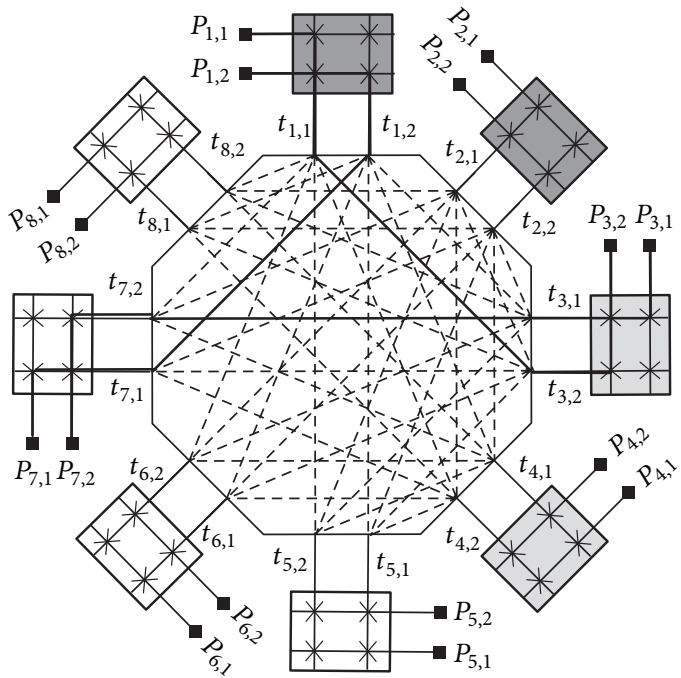

(b)

FIGURE 11: Instances of an AS routable on a $\operatorname{PSN}_{\mathrm{SU}}(2,2,2,2,4)$.

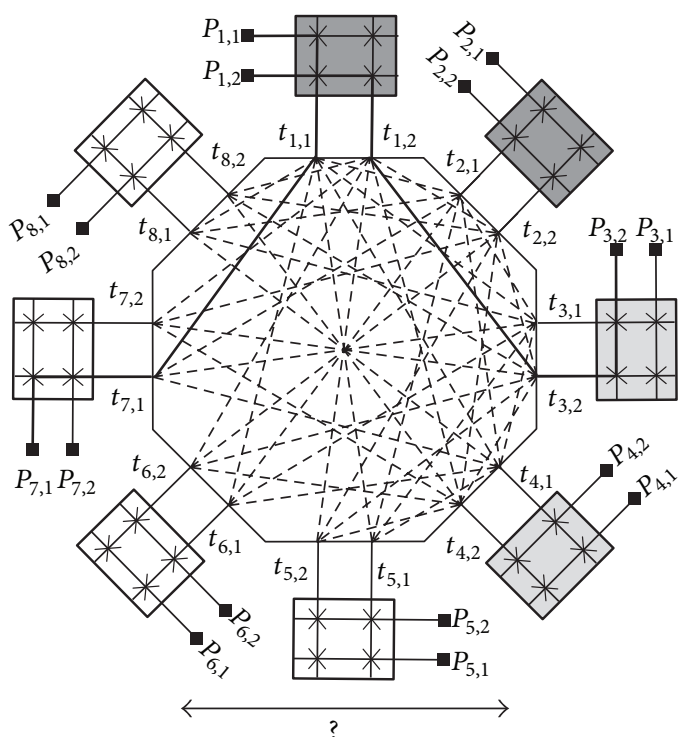

(a)

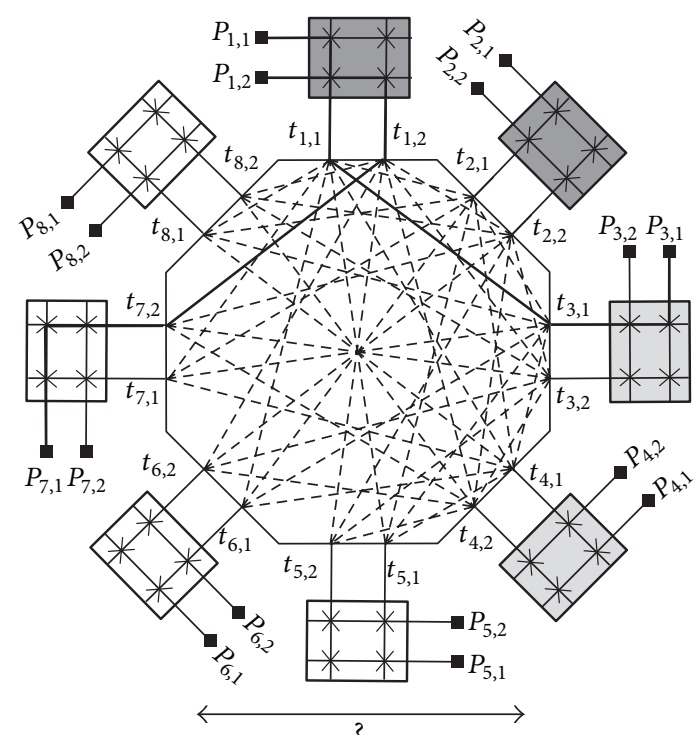

(b)

FIGURE 12: Instances of the same AS not routable on a $\operatorname{PSN}_{C}(2,2,2,2,4)$.

side of $\operatorname{PSB}_{U}(m, S)$ can be interconnected to another terminal in the remaining sides. A polygonal switch block $\operatorname{PSB}(m, S)$ is said to be universal [9-11] if any RRV $\vec{r}=\left(r_{1,2}, r_{1,3}, \ldots, r_{1, S}\right.$, $\left.r_{2,3}, \ldots, r_{2, S}, \ldots, r_{2, S}, \ldots, r_{S-1, S}\right)$ satisfying the following set of inequalities can be realizable on a $\operatorname{PSB}(m, S)$, for $m \in$ even:

$$
\begin{gathered}
r_{1,1}+r_{1,2}+\cdots+r_{1, S} \leq m, \\
r_{2,1}+r_{2,2}+\cdots+r_{2, S} \leq m, \\
\vdots \\
r_{S, 1}+r_{S, 2}+\cdots+r_{S, S} \leq m,
\end{gathered}
$$

where $0 \leq r_{i, k} \leq m$ for $1 \leq i, k \leq S$, and

$$
r_{i, k}= \begin{cases}r_{k, i}, & \text { for } 1 \leq i \neq k \leq S \\ 0, & \text { for } 1 \leq i=k \leq S\end{cases}
$$

Note that the number of connections interconnecting through each side of $\operatorname{PSB}(m, S)$ cannot exceed $m$; this dimensional constraint [9-11] is characterized by the preceding $S$ inequalities, one for each side.

Label the terminals on the $i$ th side of a $\operatorname{PSB}(m, S)$ as $T_{i}=$ $\left\{t_{i, 1}, t_{i, 2}, \ldots, t_{i, m}\right\}$ for $1 \leq i \leq S$. A generic universal switch block has been proposed by Shyu et al. [11]. As shown in 


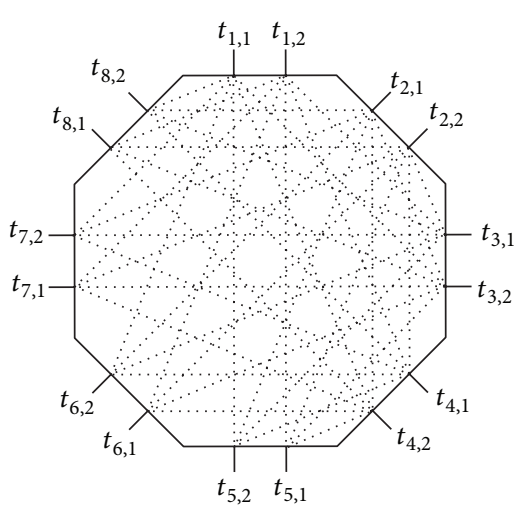

(a)

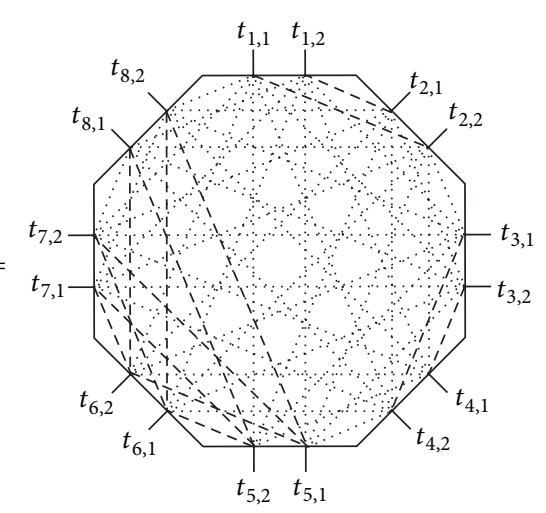

(b)

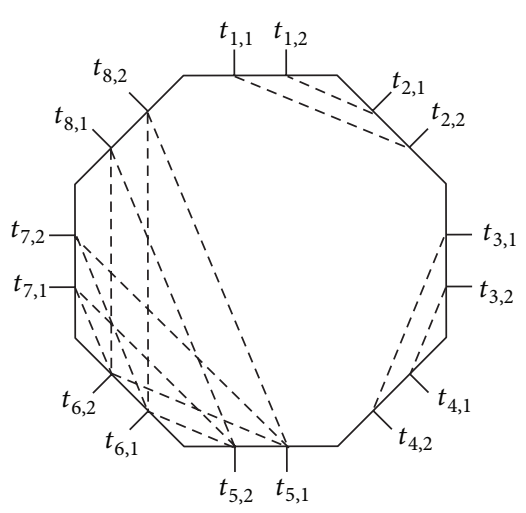

(c)

FIGURE 13: Semiuniversal and universal polygonal switch block. (a) A semiuniversal $\operatorname{PSB}_{\mathrm{SU}}(2,2,2,4)$ and (b) its equivalent to a universal $\operatorname{PSB}_{U}(2,8)$ minus some switches in (c).

Algorithm 3, the algorithm can be used to construct an $S$ side universal switch block $\operatorname{PSB}_{U}(m, S)$ with $m$ terminals on each side. That is to say, any RRV $\vec{r}$ satisfying the dimension constraint (i.e., the number of connections on each side of a $\operatorname{PSB}(m, S)$ is at most $m)$ is realizable on a $\operatorname{PSB}_{U}(m, S)$, for $S \geq 2$ and $m \in$ even [12]. For example, Figure 13(b) shows a universal $\operatorname{PSB}_{U}(2,6)$.

Since a semiuniversal $\operatorname{PSB}_{\mathrm{SU}}\left(m, s_{p}, s_{o}, s_{i}\right)$ in a $\mathrm{PSN}_{\mathrm{SU}}$ $\left(n, m, s_{p}, s_{o}, s_{i}\right)$ does not allow any two internal terminals in the same set of $T_{P}, T_{O}$, and $T_{I}$ to be interconnected, where $T_{P}=T_{1} \cup T_{2} \cup \cdots \cup T_{s_{p}}, T_{O}=T_{s_{p+1}} \cup T_{s_{p+2}} \cup \cdots \cup$ $T_{s_{p}+s_{o}}$, and $T_{I}=T_{s_{p}+s_{o}+1} \cup T_{s_{p}+s_{o}+2} \cup \cdots \cup T_{s_{p}+s_{o}+s_{i}}$, we have to modify Algorithm 3 to construct our $\operatorname{PSB}_{\mathrm{SU}}\left(m, s_{p}, s_{o}, s_{i}\right)$. Algorithm 2 removes the switches $\operatorname{SW}\left(t_{i, j}, t_{k, l}\right)$, where $t_{i, j}$ and $t_{k, l}$ belong to the same set of $T_{P}, T_{O}$, and $T_{I}$ from $\operatorname{PSB}_{U}(m, S), S=s_{p}+s_{o}+s_{i}$. For example, Figure 13(a) shows a semiuniversal switch block $\operatorname{PSB}_{\mathrm{SU}}(2,2,2,4)$, which is equal to a universal $\operatorname{PSB}_{U}(2,8)$ in Figure $13(\mathrm{~b})$ minus some prohibited switches in Figure 13(c). That is to say, a semiuniversal switch block $\operatorname{PSB}_{\mathrm{SU}}\left(m, s_{p}, s_{o}, s_{i}\right)$ contains a partial topology of the universal switch block $\operatorname{PSB}_{U}(m, S)$.

In the following, we redefine the properties of the semiuniversal and polygonal switch block $\operatorname{PSB}_{\mathrm{SU}}\left(m, s_{p}, s_{o}, s_{i}\right)$. A polygonal switch block $\operatorname{PSB}\left(m, s_{p}, s_{o}, s_{i}\right)$ is said to be Semiuniversal (SU) if any RRV $\vec{r}=\left(r_{1, s_{p}+1}, r_{1, s_{p}+2}\right.$, $\left.\ldots, r_{1, S}, r_{2, s_{p}+1}, \ldots, r_{2, S}, \ldots, r_{s_{p}, s_{p}+s_{o}+1}, \ldots, r_{s_{p}, S}, \quad \ldots, r_{s_{p}+s_{o}, S}\right)$ satisfying the following set of inequalities is realizable on a $\operatorname{PSB}\left(m, s_{p}, s_{o}, s_{i}\right)$ :

$$
\begin{gathered}
r_{1, s_{p}+1}+r_{1, s_{p}+2}+\cdots+r_{1, S} \leq m, \\
r_{2, s_{p}+1}+r_{2, s_{p}+2}+\cdots+r_{2, S} \leq m, \\
\vdots \\
r_{S, 1}+r_{S, 2}+\cdots+r_{S, s_{p}+s_{o}} \leq m,
\end{gathered}
$$

where $0 \leq r_{i, k}=r_{k, i} \leq m, m \in$ even, $S=s_{p}+s_{o}+s_{i}$ for $1 \leq i, k \leq S$, and $T_{i}$ and $T_{k}$ belong to two different sets of $T_{P}$, $T_{O}$, and $T_{I}$.
Note that the number of connections interconnecting through each side of $\operatorname{PSB}\left(m, s_{p}, s_{o}, s_{i}\right)$ cannot exceed $m$; this dimensional constraint is characterized by the preceding $S$ inequalities, one for each side. Thus, the $\operatorname{PSB}_{\mathrm{SU}}\left(m, s_{p}, s_{o}, s_{i}\right)$ constructed by Algorithm 2 is semiuniversal. Also, any RRV $\vec{r}$ satisfying the dimension constraint is simultaneously realizable on a $\operatorname{PSB}_{\mathrm{SU}}\left(m, s_{p}, s_{o}, s_{i}\right)$.

\section{Rearrangeable 3-Sided Switching Networks}

Since the programmable switches usually have high resistance and capacitance and occupy a large area, a rearrangeable 3-sided switching network PSN has routability and area efficiency. A 3-sided switching network $\operatorname{PSN}_{\mathrm{SU}}\left(n, m, s_{p}, s_{o}, s_{i}\right)$ can be constructed with $S \mathrm{CB}(n, m)$ crossbars and a semiuniversal $\operatorname{PSB}_{\mathrm{SU}}\left(m, s_{p}, s_{o}, s_{i}\right)$. Based on the semiuniversality of a $\operatorname{PSB}_{\mathrm{SU}}\left(m, s_{p}, s_{o}, s_{i}\right)$ and the properties of a crossbar $\mathrm{CB}(n, m)$, we proceed to prove that the constructed $\operatorname{PSN}_{\mathrm{SU}}\left(n, m, s_{p}, s_{o}, s_{i}\right)$ is rearrangeable.

Theorem 2. A 3-sided switching network $\operatorname{PSN}_{S U}\left(n, m, s_{p}, s_{o}\right.$, $s_{i}$ ) is rearrangeable if and only if $m \geq n, m \in$ even, $S=s_{p}+$ $s_{o}+s_{i}, s_{p} \geq 1, s_{o} \geq 1$, and $s_{i} \geq 1$.

Proof. Observably, if a $\operatorname{PSN}_{\mathrm{SU}}\left(n, m, s_{p}, s_{o}, s_{i}\right)$ with $m=n$ is rearrangeable, then a $\operatorname{PSN}_{\mathrm{SU}}\left(n, m, s_{p}, s_{o}, s_{i}\right)$ with $m \geq n$ is rearrangeable. Thus, we need to prove that a $\operatorname{PSN}_{\mathrm{SU}}\left(n, n, s_{p}\right.$, $s_{o}, s_{i}$ ) is rearrangeable, $n \in$ even.

A $\operatorname{PSN}_{\mathrm{SU}}\left(n, n, s_{p}, s_{o}, s_{i}\right)$ is used to connect the external terminals of $P_{P}, P_{O}$, and $P_{I}$ to each other. Let $P_{P}=P_{1} \cup P_{2} \cup$ $\cdots \cup P_{s_{p}}$ (I/O terminals), $P_{O}=P_{s_{p+1}} \cup P_{s_{p+2}} \cup \cdots \cup P_{s_{p}+s_{o}}$ (output terminals), and $P_{I}=P_{s_{p}+s_{o}+1} \cup P_{s_{p}+s_{o}+2} \cup \cdots \cup P_{s_{p}+s_{o}+s_{i}}$ (input terminals), where $P_{i}=\left\{p_{i, 1}, p_{i, 2}, \ldots, p_{i, n}\right\}$ on a $\mathrm{CB}_{i}(n, n)$, $1 \leq i \leq S$. The connection pair $\left(p_{i, j}, p_{k, l}\right)$ is a point-to-point connection, where $p_{i, j}$ and $p_{k, l}$ belong to different sets of $P_{P}$, $P_{O}$, and $P_{I}$. An assignment AS $=\left\{\left(p_{i, j}, p_{k, l}\right)\right\}$ represents a set of connection pairs to be connected; thus each external terminal appears in at most one pair. The $\operatorname{PSN}_{\mathrm{SU}}\left(n, n, s_{p}, s_{o}, s_{i}\right)$ is rearrangeable if any assignment AS is realizable. 


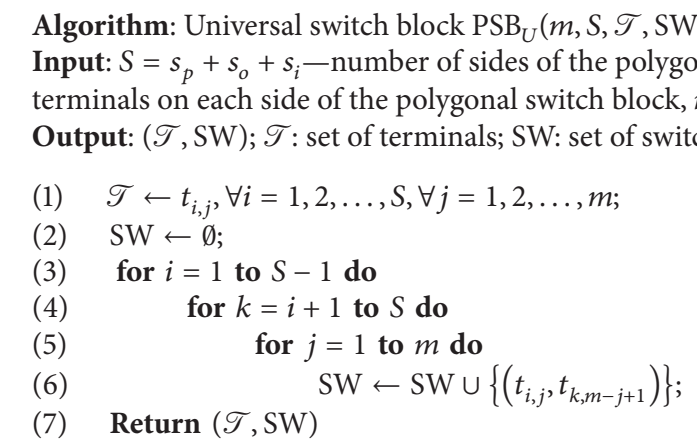

Algorithm 3: Algorithm for constructing an $S$-sided universal switch block of size $m$.

(If) Any assignment $\mathrm{AS}=\left\{\left(p_{i, j}, p_{k, l}\right)\right\}$ can be connected on $\operatorname{PSN}_{\mathrm{SU}}\left(n, n, s_{p}, s_{o}, s_{i}\right)$, since each connection pair $\left(p_{i, j}, p_{k, l}\right) \in \mathrm{AS}$ is required to be connected by passing $\mathrm{CB}_{i}-\mathrm{PSB}-\mathrm{CB}_{k}$, we can obtain that this kind of RRV $\vec{r}=$ $\left(r_{1, s_{p}+1}, r_{1, s_{p}+2}, \ldots, r_{1, s_{p}+s_{o}+s_{i}}, r_{2, s_{p}+1}, \ldots, r_{2, s_{p}+s_{o}+s_{i}}, \ldots, r_{s_{p}, s_{p}+1}\right.$, $\left.\ldots, r_{s_{p}, s_{p}+s_{o}+s_{i}}, \ldots, r_{s_{p}+s_{o}, s_{p}+s_{o}+s_{i}}\right)$ for any given AS to be realized on a $\operatorname{PSN}_{\mathrm{SU}}\left(n, s_{p}, s_{o}, s_{i}\right)$, where $r_{i, k}$ represents the number of type- $(i, k)$ connections between $P_{i}$ and $P_{k}$. Furthermore, this RRV $\vec{r}$ satisfying the dimension constraint is realizable on a semiuniversal $\operatorname{PSB}_{\mathrm{SU}}\left(n, s_{p}, s_{o}, s_{i}\right)$, due to the number of connection pairs in AS interconnecting through each side of $\operatorname{PSB}_{\mathrm{SU}}\left(n, s_{p}, s_{o}, s_{i}\right)$ does not exceed $n$. Therefore, for each $\left(p_{i, j}, p_{k, l}\right) \in$ AS, we can find a connection path $\left(t_{i, q}, t_{k, n-q+1}\right)$ between $T_{i}$ and $T_{k}$ after the RRV $\vec{r}$ has been realized on a $\operatorname{PSB}_{\mathrm{SU}}\left(n, s_{p}, s_{o}, s_{i}\right)$, where $1 \leq q \leq n$. Then the connection $\left(p_{i, j}, p_{k, l}\right)$ is realizable by programmed three switches, one switch $\operatorname{SW}\left(t_{i, q}, t_{k, n-q+1}\right)$ in the $\operatorname{PSB}_{\mathrm{SU}}\left(n, s_{p}, s_{o}, s_{i}\right)$ and two other switches $\operatorname{SW}\left(p_{i, j}, t_{i, q}\right)$ and $\operatorname{SW}\left(p_{k, l}, t_{k, n-q+1}\right)$ in the $\mathrm{CB}_{i}(n, n)$ and $\mathrm{CB}_{k}(n, n)$, respectively. Therefore, any given assignment $\mathrm{AS}$ for a $\operatorname{PSN}_{\mathrm{SU}}\left(n, n, s_{p}, s_{o}, s_{i}\right)$ is realizable, because there exist in the $\operatorname{PSN}_{\mathrm{SU}}\left(n, n, s_{p}, s_{o}, s_{i}\right)$ disjoint paths connecting all the pairs of terminals given in the assignment AS. Thus, a $\operatorname{PSN}_{\mathrm{SU}}\left(n, m, s_{p}, s_{o}, s_{i}\right)$ switching networks with $m \geq n$ is rearrangeable.

(Only if) If in a $\operatorname{PSN}_{\mathrm{SU}}\left(n, m, s_{p}, s_{o}, s_{i}\right)$ with $m<n$, we have an assignment $\mathrm{AS}=\left\{\left(p_{i, 1}, p_{k, 1}\right),\left(p_{i, 2}, p_{k, 2}\right), \ldots\right.$, $\left.\left(p_{i, n}, p_{k, n}\right)\right\}$ to be connected between the $P_{i}$ and $P_{k}$ on a $\operatorname{PSN}_{\mathrm{SU}}\left(n, m, s_{p}, s_{o}, s_{i}\right)$, where $P_{i}$ and $P_{k}$ belong to different sets of $P_{P}, P_{O}$, and $P_{I}$, since each connection pair $\left(p_{i, j}, p_{k, l}\right) \in$ AS is connected by passing blocks $\mathrm{CB}_{i}-\mathrm{PSB}-\mathrm{CB}_{k}$. In each $\mathrm{CB}_{i}(n, m)$, we cannot find enough disjoint paths to connect all the $n$ external terminals in $P_{i}$ to all the $m$ internal terminals in $T_{i}$, which in turn are connected to the $i$ th side of a $\operatorname{PSB}_{\mathrm{SU}}\left(m, s_{p}, s_{o}, s_{i}\right)$, due to $m<n$. Thus, this AS is not realizable on a $\operatorname{PSN}_{\mathrm{SU}}\left(n, m, s_{p}, s_{o}, s_{i}\right)$ with $m<n$.

\section{Minimize the Number of Switches}

We have shown a rearrangeable 3-sided switching network $\operatorname{PSN}_{\mathrm{SU}}\left(n, n, s_{p}, s_{o}, s_{i}\right)$ in the above section. Now, we start to explore the effect of the parameters $s_{p}, s_{o}, s_{i}$, and $n$ in a $\operatorname{PSN}_{\mathrm{SU}}\left(n, n, s_{p}, s_{o}, s_{i}\right)$ on the switch-efficiency, and we try to find proper $s_{p}, s_{o}, s_{i}$, and $n$ values to minimize the number of switches needed in a rearrangeable $\operatorname{PSN}_{\mathrm{SU}}\left(n, n, s_{p}, s_{o}, s_{i}\right)$ to interconnect terminal pairs from the three sets of $P_{P}, P_{O}$, and $P_{I}$. We assume that the set of $P_{P}, P_{O}$, and $P_{I}$ have $N_{P}=s_{p} \times n$, $N_{O}=s_{o} \times n$, and $N_{I}=s_{i} \times n$ external terminals, respectively.

Since the number of switches in $\operatorname{SCB}(n, n)$ crossbars is equal to $S n^{2}=\left(s_{p}+s_{o}+s_{i}\right) n^{2}$ and the number of switches in a polygonal switch block $\operatorname{PSB}_{\mathrm{SU}}\left(n, s_{p}, s_{o}, s_{i}\right)$ is equal to $n\left(s_{p} s_{o}+s_{p} s_{i}+s_{o} s_{i}\right)$, we denote the number of switches in a $\operatorname{PSN}_{\mathrm{SU}}\left(n, n, s_{p}, s_{o}, s_{i}\right)$ as $\operatorname{SW}_{\mathrm{SU}}\left(n, n, s_{p}, s_{o}, s_{i}\right)$. By summing the number of switches in all the above blocks, we have

$$
\begin{aligned}
\mathrm{SW}_{\mathrm{SU}}\left(n, n, s_{p}, s_{o}, s_{i}\right)= & \left(s_{p}+s_{o}+s_{i}\right) n^{2} \\
& +n\left(s_{p} s_{o}+s_{p} s_{i}+s_{o} s_{i}\right) .
\end{aligned}
$$

Substituting $s_{p}=N_{P} / n, s_{o}=N_{O} / n$, and $s_{i}=N_{I} / n$ into (4) results in

$$
\begin{aligned}
\operatorname{SW}_{\mathrm{SU}}\left(n, n, s_{p}, s_{o}, s_{i}\right)= & n\left(N_{P}+N_{O}+N_{I}\right) \\
& +\frac{N_{P} N_{O}+N_{P} N_{I}+N_{O} N_{I}}{n} .
\end{aligned}
$$

Let $d \mathrm{SW} / d n=0$ and solve for $n$, since $N_{P}, N_{O}$, and $N_{I}$ are constant, we have

$$
\begin{aligned}
\frac{d \mathrm{SW}_{\mathrm{SU}}\left(n, n, s_{p}, s_{o}, s_{i}\right)}{d n}= & \left(N_{P}+N_{O}+N_{I}\right) \\
& -\frac{N_{P} N_{I}+N_{P} N_{O}+N_{O} N_{I}}{n^{2}}=0,
\end{aligned}
$$

which implies

$$
\begin{gathered}
n=\sqrt{\frac{N_{P} N_{I}+N_{P} N_{O}+N_{O} N_{I}}{N_{P}+N_{O}+N_{I}}}, \quad s_{p}=\frac{N_{P}}{n}, \\
s_{o}=\frac{N_{O}}{n}, \quad s_{i}=\frac{N_{I}}{n}, \quad \frac{d^{2} \mathrm{SW}}{d n^{2}}>0 .
\end{gathered}
$$


TABLE 2: Number of switches needed in five rearrangeable $\operatorname{PSN}_{\mathrm{SU}}\left(n, n, s_{p}, s_{o}, s_{i}\right)$ s for interconnecting terminal pair from three terminal sets, where $N_{P}=64, N_{O}=32$, and $N_{I}=128$ terminals.

\begin{tabular}{lrccc}
\hline \multicolumn{3}{c}{$\mathrm{PSN}_{\mathrm{SU}}$} & \multicolumn{2}{c}{$\mathrm{SW}_{\mathrm{SU}}\left(n, n, s_{p}, s_{o}, s_{i}\right)$} \\
\hline 2 & $s_{p}$ & $s_{o}$ & $s_{i}$ & 7616 \\
4 & 32 & 16 & 64 & 4480 \\
$\mathbf{8}$ & 16 & 8 & 32 & $\mathbf{3 5 8 4}$ \\
16 & $\mathbf{8}$ & $\mathbf{4}$ & $\mathbf{1 6}$ & 4480 \\
32 & 4 & 2 & 8 & 7616 \\
\hline
\end{tabular}

TABLE 3: Number of switches needed in five rearrangeable $\operatorname{PSN}_{\mathrm{SU}}\left(n, n, s_{p}, s_{o}, s_{i}\right)$ s for interconnecting terminal pair from three terminal sets, where $N_{P}=144, N_{O}=96$, and $N_{I}=192$ terminals.

\begin{tabular}{lcccc}
\hline & \multicolumn{2}{c}{ PSN $_{\mathrm{SU}}$} & & $\mathrm{SW}_{\mathrm{SU}}\left(n, n, s_{p}, s_{o}, s_{i}\right)$ \\
\hline 2 & $s_{p}$ & $s_{o}$ & $s_{i}$ & 30816 \\
4 & 72 & 48 & 96 & 16704 \\
6 & 36 & 24 & 48 & 12576 \\
8 & 24 & 16 & 32 & 10944 \\
$\mathbf{1 2}$ & 18 & 12 & 24 & $\mathbf{1 0 1 7 6}$ \\
24 & $\mathbf{1 2}$ & $\mathbf{8}$ & $\mathbf{1 6}$ & 12864 \\
48 & 6 & 4 & 8 & 21984 \\
\hline
\end{tabular}

This indicates that the function $\mathrm{SW}_{\mathrm{SU}}\left(n, n, s_{p}, s_{o}, s_{i}\right)$ has minimum value at $n=$ $\sqrt{\left(N_{P} N_{I}+N_{P} N_{O}+N_{O} N_{I}\right) /\left(N_{P}+N_{O}+N_{I}\right)}$,

$$
\begin{aligned}
& \mathrm{SW}_{\mathrm{SU}}\left(n, n, s_{p}, s_{o}, s_{i}\right) \\
& \quad=2 \sqrt{\left(N_{P}+N_{O}+N_{I}\right)\left(N_{P} N_{I}+N_{P} N_{O}+N_{O} N_{I}\right)} .
\end{aligned}
$$

From (8), we have that a $\operatorname{PSN}_{\mathrm{SU}}\left(n, n, s_{p}, s_{o}, s_{i}\right)$ with $n=\sqrt{\left(N_{P} N_{I}+N_{P} N_{O}+N_{O} N_{I}\right) /\left(N_{P}+N_{O}+N_{I}\right)}$ contains the number of switches to a minimum. Given a $\operatorname{PSN}_{\mathrm{SU}}\left(n, n, s_{p}, s_{o}, s_{i}\right)$ interconnecting terminals pairs from $P_{P}, P_{O}$, and $P_{I}$, where the set of $P_{P}, P_{O}$, and $P_{I}$ have $N_{P}$, $N_{O}$, and $N_{I}$ external terminals, respectively. Tables 2 and 3 show the number of switches needed in rearrangeable $\operatorname{PSN}_{\mathrm{SU}}\left(n, n, s_{p}, s_{o}, s_{i}\right)$ each of which varies with $n$. In Table 2 , the rearrangeable switching network $\operatorname{PSN}_{\mathrm{SU}}(8,8,8,4,16)$ with $n=8$ (from (8)) is the best choice for interconnecting terminals pairs from the three terminal sets in terms of the number of switches. Furthermore, in Table 3, the rearrangeable switching network $\operatorname{PSN}_{\mathrm{SU}}(12,12,12,8,16)$ with $n=11.7$ (from (8)) is the best choice for interconnecting terminals pairs from the three terminal sets.

\section{Polygonal FPGA}

Figure 2 shows a model of polygonal FPGA (PFPGA), which consists of many clusters of logic block and $I / O$ pins interconnected by a three-sided polygonal switching network. In this study, we investigate the logic block based on a cluster of 4-input look-up tables (LUTs). The three-sided polygonal

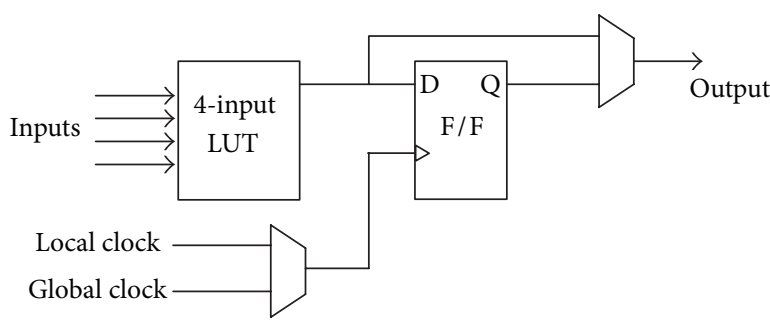

FIGURE 14: Basic logic block.

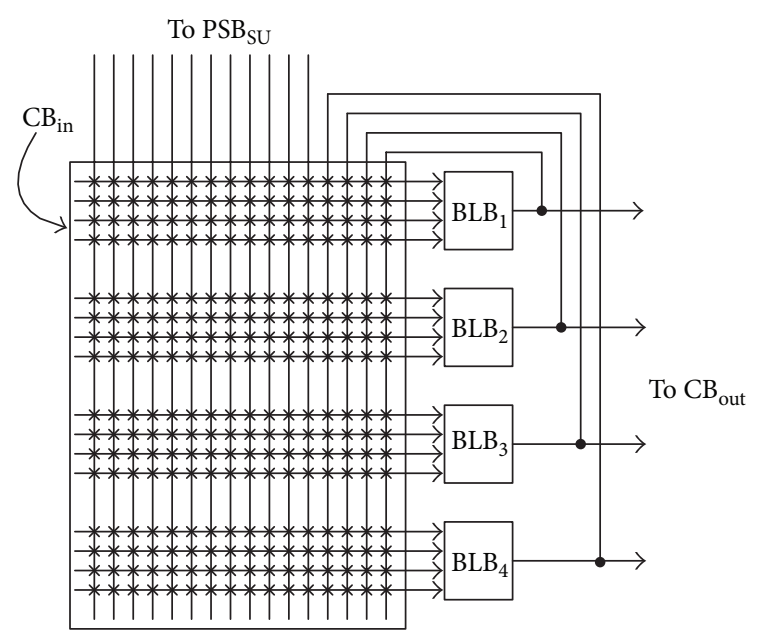

FIGURE 15: Cluster-based logic block.

switching network consists of $S$ connection blocks (CBs) and an $S$-sided polygonal switch block (PSB). Each connection block is connected to one of the $S$ sides of the polygonal switch block. All the above blocks contain many programmable switches. The polygonal switching network is a three-stage three-sided switching network [4] for connecting inputs of logic-block, outputs of logic-block, and $I / O$ pins to interconnect each other. First, the input and output pins of clusterbased logic blocks and $I / O$ pins are connected to the related connection blocks (CBs), which are then interconnected by a three-sided polygonal switch block (PSB). That is, the first and third stages consist of $S$ CBs and the second stage is a PSB with $S$ sides. In this PFPGA, any two terminals of the three groups in a PFPGA can be connected with fewer than three switches. Thus, the speed performance of a PFPGA can be improved.

Figure 14 shows that the basic logic block (BLB) [18] of our PFPGA has $r=5$ inputs and 1 output excluding the "global clock". A BLB consists of a 4-input LUT and a register, and the BLB output can be either the registered or unregistered version of the LUT output. A BLB contains configurable digital circuits that can be used to implement different logic functions. As shown in Figure 15, the complete cluster-based logic block (CLB) is comprised of $k \mathrm{BLB}$, denoted as $\operatorname{CLB}(k)$. $\mathrm{A} \mathrm{CLB}(k)$ has $r k$ inputs and $k$ outputs. Let $n=r k$ on a $\mathrm{CB}(n, m)$; then $r k$ inputs of a $\operatorname{CLB}(k)$ are connected to one $\mathrm{CB}(n, m)$ of $\mathrm{CB}_{I}$, and $r k$ outputs of $r \mathrm{CLB}(k)$ 's connected to one $\mathrm{CB}(n, m)$ of $\mathrm{CB}_{O}$. Let $L$ be the 
total number of BLB's in a PFPGA with $B I / O$ pins, as shown in Figure 2. We can obtain the following three parameters $s_{i}$, $s_{o}$, and $s_{p}$ for a PFPGA:

$$
\begin{gathered}
s_{i}=\left\lceil\frac{L}{k}\right\rceil, \quad s_{o}=\left\lceil\frac{L}{n}\right\rceil, \quad s_{p}=\left\lceil\frac{B}{n}\right\rceil, \\
S=s_{i}+s_{o}+s_{p}, \quad n=r k .
\end{gathered}
$$

The key factors to the PFPGA switch-efficiency and speed-efficiency are the structure of its switching network $\operatorname{PSN}_{\mathrm{SU}}\left(n, m, s_{p}, s_{o}, s_{i}\right)$ structure and the granularity of its cluster-based logic blocks $\operatorname{CLB}(k)$. That is to say, the parameters $k$ and $m$ in a PFPGA affect its speed performance, die size, and routability.

Cluster-based logic blocks have two other advantages over single BLB logic blocks. First, in an FPGA composed of logic clusters, many nets will be completely contained within a logic cluster. If these nets are routed using multiplexers within a cluster, the delay of these multiplexers is less than that of the main FPGA. Secondly, clustering $k$ BLB's into one logic cluster will greatly reduce the placement time by a factor of $k$, which is of increasing concern in today's large FPGA's.

5.1. Number of Switches in a $P S N_{S U}$. Let $L$ be the total number of BLB's in a PFPGA with $B I / O$ pins, and a BLB has $r$ inputs and 1 output. The complete cluster-based logic block (CLB) is composed of $k$ BLB's, denoted as $\operatorname{CLB}(k)$. The number of switches in polygonal switching network $\operatorname{PSN}_{\mathrm{SU}}\left(n, m, s_{p}, s_{o}, s_{i}\right)$ is obtained as follows.

Because we have the following facts:

(i) the number of switches in $S \mathrm{CB}(n, m)$ 's is equal to $S \times$ $m \times n$,

(ii) the number of switches in a $\operatorname{PSN}_{\mathrm{SU}}\left(m, s_{p}, s_{o}, s_{i}\right)$ is equal to $m\left(s_{p} s_{o}+s_{p} s_{i}+s_{o} s_{i}\right)$, where $n=r k, s_{i}=\lceil L / k\rceil$, $s_{o}=\lceil L / n\rceil, s_{p}=\lceil B / n\rceil$, and $S=s_{i}+s_{o}+s_{p}$, and $L, B$ and $r$ are constants.

Denote the number of switches in a $\operatorname{PSN}_{\mathrm{SU}}\left(n, m, s_{p}, s_{o}, s_{i}\right)$ as $\mathrm{SW}_{\mathrm{SU}}\left(n, m, s_{p}, s_{o}, s_{i}\right)$. By summing the number of switches in all the above blocks, we have

$$
\mathrm{SW}_{\mathrm{SU}}\left(n, m, s_{p}, s_{o}, s_{i}\right)=\operatorname{Smn}+m\left(s_{p} s_{o}+s_{p} s_{i}+s_{o} s_{i}\right)
$$

From (10), $\operatorname{SW}_{\mathrm{SU}}\left(n, m, s_{p}, s_{o}, s_{i}\right)$ is determined by the $k$ and $m$ values. In the following section, we will find proper $k$ values and $m$ to minimize the number of switches needed in a polygonal switching network through experiments.

5.2. Experimental Results. To explore the effects of $k$ and $m$ values of a polygonal switching network on the switchefficiency in a PFPGA with $L$ BLB's and $B I / O$ pins, we have implemented a maze router [19-21] in $C$ language and executed the codes on an IBM System M3. We examine the effect of two parameters, $k$ and $m$, on the switch performance using the CGE [1] and SEGA [22] benchmark circuits.

By routing different cluster size of logic blocks, the switch performance of a three-sided polygonal switching network
TABLE 4: Minimum number of tracks and switches needed for detailed-routing completion for CLB(5).

\begin{tabular}{lccccccc}
\hline \multirow{2}{*}{ Circuit } & \multirow{2}{*}{ LBs } & \multirow{2}{*}{ \#Con. } & \multicolumn{5}{c}{$\mathrm{PSN}_{\mathrm{SU}}\left(n, m, s_{i}, s_{o}, s_{p}\right)$} \\
& & & $m$ & $s_{i}$ & $s_{o}$ & $s_{p}$ & $\mathrm{SW}_{\mathrm{SU}}$ \\
\hline BUSC & $24 \times 22$ & 392 & 22 & 22 & 6 & 4 & 9064 \\
DMA & $34 \times 30$ & 771 & 24 & 45 & 11 & 2 & 14358 \\
DFSM & $44 \times 42$ & 1422 & 27 & 84 & 20 & 3 & 30690 \\
BNRE & $42 \times 40$ & 1257 & 27 & 76 & 19 & 4 & 29517 \\
Z03 & $52 \times 50$ & 2135 & 27 & 120 & 29 & 3 & 42894 \\
\hline 9symml & $20 \times 18$ & 259 & 22 & 15 & 4 & 1 & 4428 \\
alu2 & $28 \times 24$ & 511 & 23 & 29 & 7 & 1 & 8007 \\
alu4 & $36 \times 32$ & 851 & 27 & 51 & 13 & 2 & 17235 \\
apex7 & $22 \times 18$ & 300 & 22 & 16 & 4 & 5 & 7988 \\
example2 & $26 \times 22$ & 444 & 23 & 24 & 6 & 8 & 13122 \\
k2 & $42 \times 38$ & 1256 & 25 & 72 & 18 & 5 & 28050 \\
term1 & $18 \times 16$ & 202 & 22 & 12 & 3 & 3 & 5100 \\
too_large & $28 \times 26$ & 519 & 22 & 32 & 8 & 3 & 11004 \\
vda & $32 \times 30$ & 722 & 25 & 42 & 10 & 3 & 14750 \\
\hline Total & - & - & - & - & - & - & 236207 \\
\hline
\end{tabular}

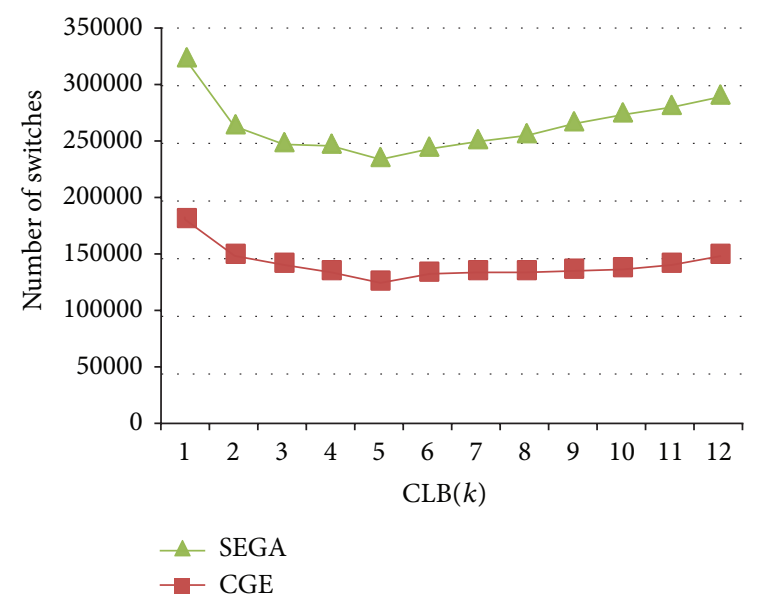

FIGURE 16: Minimum number of switches needed for 14 benchmark circuits.

was evaluated. From the results of a $\operatorname{CLB}(5)$, as listed in Table 4, we first determine the minimum number of tracks $m$ required to complete the $100 \%$ routing of each circuit, using three-sided polygonal switching networks $\operatorname{PSN}_{\mathrm{SU}}\left(n, m, s_{p}\right.$, $\left.s_{o}, s_{i}\right)$. Then we can find the $\mathrm{SW}_{\mathrm{SU}}\left(n, m, s_{p}, s_{o}, s_{i}\right)$ value by substituting the values of $n, m, s_{i}, s_{o}$, and $s_{p}$ into (10).

Figure 16 shows that the numbers of programmable switches $\mathrm{SW}_{\mathrm{SU}}\left(n, m, s_{p}, s_{o}, s_{i}\right)$ used in the 14 benchmarks vary with $\operatorname{CLB}(k)$, where $k=1,2, \ldots, 12$. Experimental results demonstrate that the switches number used in $\mathrm{PSN}_{\mathrm{SU}}$ is minimum for a PFPGA with $\mathrm{CLB}(5)$ (for CGE) and CLB(3) (for SEGA), respectively. We assume that the available pin count of a PFPGA is $B=84$ and the number of BLB is about 208. Therefore, our PFPGA consists of $52 \mathrm{CLB}(5)$ and $B=84 \mathrm{I} / \mathrm{O}$ pins interconnected by a three-sided polygonal 


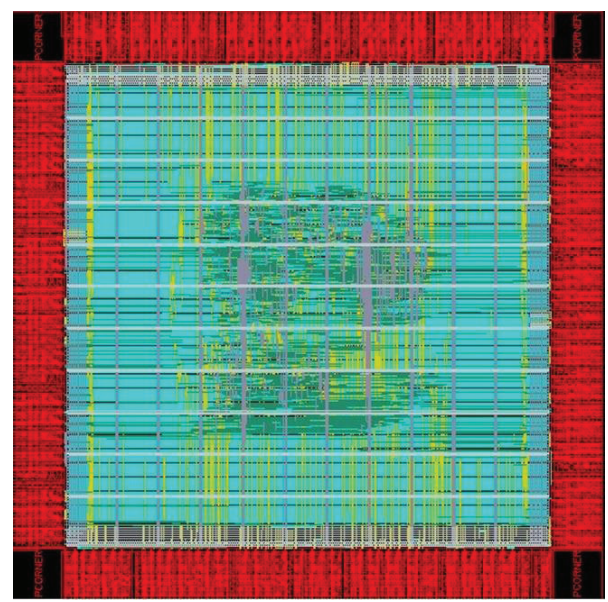

FIGURE 17: Chip layout of the PFPGA.

switching network $\operatorname{SW}_{\mathrm{SU}}\left(n=20, m=25, s_{i}=52, s_{o}=11\right.$, $\left.s_{p}=5\right)$.

5.3. VLSI Chip Implementation of the PFPGA. The chip layout of our PFPGA using a $0.18 \mu \mathrm{m}$ CMOS technology is shown in Figure 17. The polygonal switching network area of a PFPGA is $1255 \mu \mathrm{m} \times 1350 \mu \mathrm{m}$. And the total area of this PFPGA is $1550 \mu \mathrm{m} \times 1600 \mu \mathrm{m}$. So we can calculate the ratio of routing area to total area to be $68.81 \%$. Since routing area typically takes 70-90 percent of the total chip area in general FPGA [3]. So we conclude that the polygonal FPGA gets more area utilization.

In our proposed PFPGA architecture, we provide a threestage routing resources concept. The first and third stages of routing are the connection blocks in two CLBs, which can meet local signal routing requirements. And the second layer of routing is the polygonal switch block, which is used to connect any output signal of a CLB to any input signal of a CLB. Thus, the longest distance in our PFPGA is only three stages. The maximum point-to-point of $\mathrm{PSN}_{\mathrm{SU}}$ delay is 13 ns. So we conclude that the architecture of PFPGA has better speed improvement. As mentioned above, this chip architecture uses less number of programmable switches and the speed performance of a PFPGA can be achieved. Thus, it is very suitable for VLSI implementation.

\section{Conclusions}

This paper first proposed a generic three-stage and rearrangeable three-sided switching network $\operatorname{PSN}_{\mathrm{SU}}\left(n, m, s_{p}, s_{o}, s_{i}\right)$ used in the PFPGA for all even $m$. The PSN consists of $S \mathrm{CB}(n, m)$ crossbars interconnected by a semiuniversal polygonal switch block $\operatorname{PSB}_{\mathrm{SU}}\left(m, s_{p}, s_{o}, s_{i}\right)$ with $S$ sides. We not only provide the designers with a rearrangeable $\operatorname{PSN}_{\mathrm{SU}}\left(n, m, s_{p}, s_{o}, s_{i}\right)$ with $m \geq n$ for connecting terminal pairs from three disjoint terminal sets to each other but also determinate the important parameter $n$ to minimize the number of switches needed in that network. We also propose a PFPGA that consists of many logic blocks interconnected by a $\mathrm{PSN}_{\mathrm{SU}}$. We investigate the effect of the $\mathrm{PSN}_{\mathrm{SU}}$ structure and the granularity of its interconnecting cluster-based logic blocks on the switch-efficiency and speedefficiency performance. Experimental results demonstrate that the switch-efficiency and speed-efficiency are improved so it holds promise as a practical PFPGA with polygonal switching network.

\section{Acknowledgment}

This work was partly supported by the National Science Council, Taiwan, under Grant NSC 101-2221-E-019-071.

\section{References}

[1] J. Rose and S. Brown, "Flexibility of interconnection structures for field-programmable gate arrays," IEEE Journal of Solid-State Circuits, vol. 26, no. 3, pp. 277-282, 1991.

[2] S. Franchini, A. Gentile, F. Sorbello, G. Vassallo, and S. Vitabile, "An embedded, FPGA-based computer graphics coprocessor with native geometric algebra support," Integration, the VLSI Journal, vol. 42, no. 3, pp. 346-355, 2009.

[3] S. D. Brown, R. J. Francis, J. Rose, and Z. G. Vranesic, FieldProgrammable Gate Arrays, Kluwer Academic, 1992.

[4] V. E. Benes, "On rearrangeable three-stage connecting networks," Bell System Technical Journal, vol. 41, no. 5, pp. 1481$1492,1962$.

[5] Y. M. Yen and T. Y. Feng, "On a class of rearrangeable networks," IEEE Transactions on Computers, vol. 41, no. 11, pp. 1361-1379, 1992.

[6] C. Mitchell and P. Wild, "One-stage one-sided rearrangeable switching networks," IEEE Transactions on Communications, vol. 37, no. 1, pp. 52-56, 1989.

[7] A. Varma and S. Chalasani, "Reduction of crosspoints in onesided crosspoint switching networks," in Proceedings of the 8th Annual Conference of the IEEE Computer and Communications Societies. Technology: Emerging or Converging? (INFOCOM '89), vol. 3, pp. 943-952, Ottawa, Canada, April 1989.

[8] J. Gordon and S. Srikanthan, "Single sided switching networks," Electronics Letters, vol. 26, no. 4, pp. 248-250, 1990.

[9] Y.-W. Chang, D. F. Wong, and C. K. Wong, "Universal switch modules for fpga design," ACM Transactions on Design Automation of Electronic Systems, vol. 1, no. 1, pp. 80-101, 1996.

[10] G. M. Wu, M. Shyu, and Y. -W. Chang, "Universal switch blocks for three-dimensional FPGA design," in Proceedings of the ACM International Symposium on Field Programmable Gate Arrays (FPGA '99), Monterey, Calif, USA, February 1999.

[11] M. Shyu, G.-M. Wu, Y.-D. Chang, and Y.-W. Chang, "Generic universal switch blocks," IEEE Transactions on Computers, vol. 49, no. 4, pp. 348-359, 2000.

[12] H. Fan, J. Liu, Y.-L. Wu, and C. K. Wong, "Reduction design for generic universal switch blocks," ACM Transactions on Design Automation of Electronic Systems, vol. 7, no. 4, pp. 526-546, 2002.

[13] M.-H. Yen, C. Yu, H.-Y. Shin, and S.-J. Chen, "A threesided rearrangeable switching network for a binary fat tree," International Journal of Electronics, vol. 98, no. 6, pp. 713-734, 2011. 
[14] M. H. Yen, M. C. Shie, and S. H. Lan, "Polygonal routing network for FPGA/FPIC," in Proceedings of the International Symposium on VLSI Technology, System, and Applications (VLSI-TSA '99), pp. 104-107, 1999.

[15] M.-H. Yen, S.-J. Chen, and S. H. Lan, "Symmetric and programmable multi-chip module for low-power prototyping system," VLSI Design, vol. 12, no. 2, pp. 113-124, 2001.

[16] M.-H. Yen, S.-J. Chen, and S. H. Lan, "A three-stage one-sided rearrangeable polygonal switching network," IEEE Transactions on Computers, vol. 50, no. 11, pp. 1291-1294, 2001.

[17] J. Rose, R. J. Francis, D. Lewis, and P. Chow, "Architecture of field-programmable gate arrays: the effect of logic block functionality on area efficiency," IEEE Journal of Solid-State Circuits, vol. 25, no. 5, pp. 1217-1225, 1990.

[18] V. Betz and J. Rose, "Cluster-based logic blocks for FPGAs: areaefficiency vs. input sharing and size," in Proceedings of the IEEE Custom Integrated Circuits Conference (CICC '97), pp. 551-554, Santa Clara, Calif, USA, May 1997.

[19] M. Marek-Sadowska, "Switch box routing: a retrospective," Integration, the VLSI Journal, vol. 13, no. 1, pp. 39-65, 1992.

[20] W. K. Luk, "A greedy switch-box router," Integration, the VLSI Journal, vol. 3, no. 2, pp. 129-149, 1985.

[21] J. Pan, Y.-L. Wu, C. K. Wong, and G. Yan, "On the optimal four-way switch box routing structures of FPGA greedy routing architectures," Integration, the VLSI Journal, vol. 25, no. 2, pp. 137-159, 1998

[22] G. G. Lemienx and S. D. Brown, "A detailed routing algorithm for allocating wire segments in field-programmable gate arrays," in Proceedings of the ACM/SIGDA Physical Design Workshop, pp. 215-216, Lake Arrowhead, Calif, USA, 1993. 

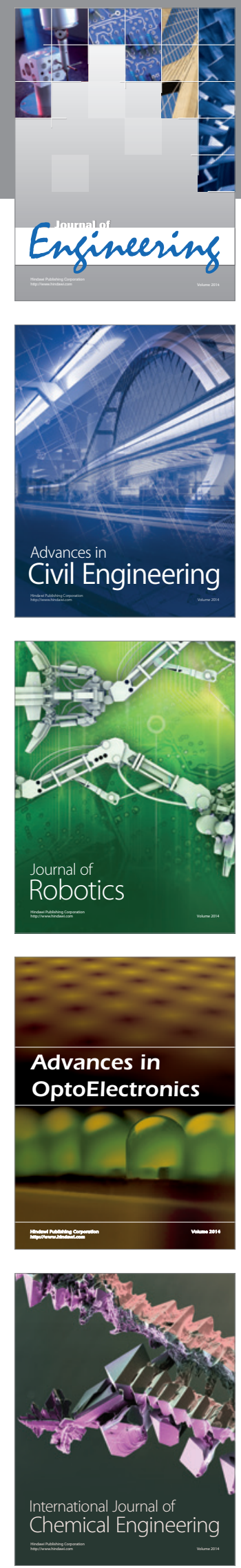

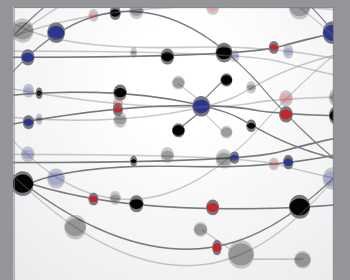

The Scientific World Journal
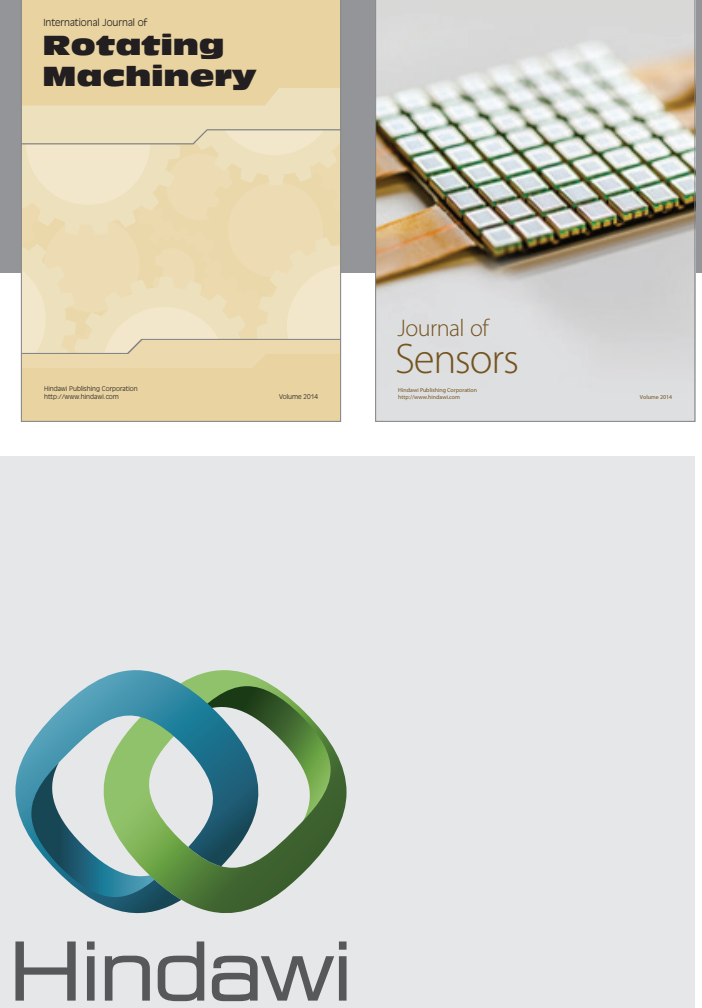

Submit your manuscripts at http://www.hindawi.com
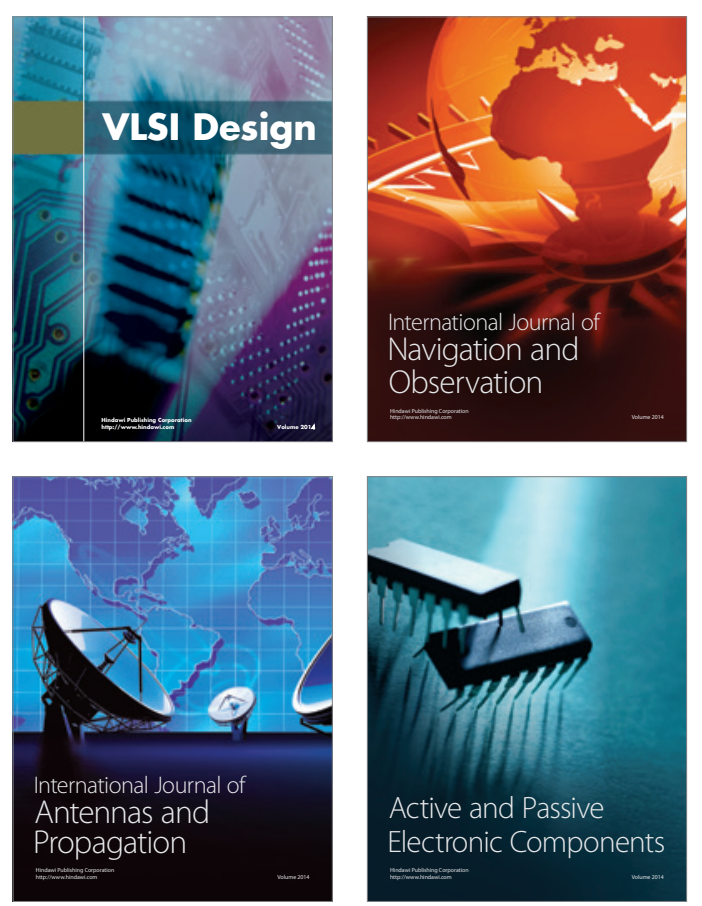
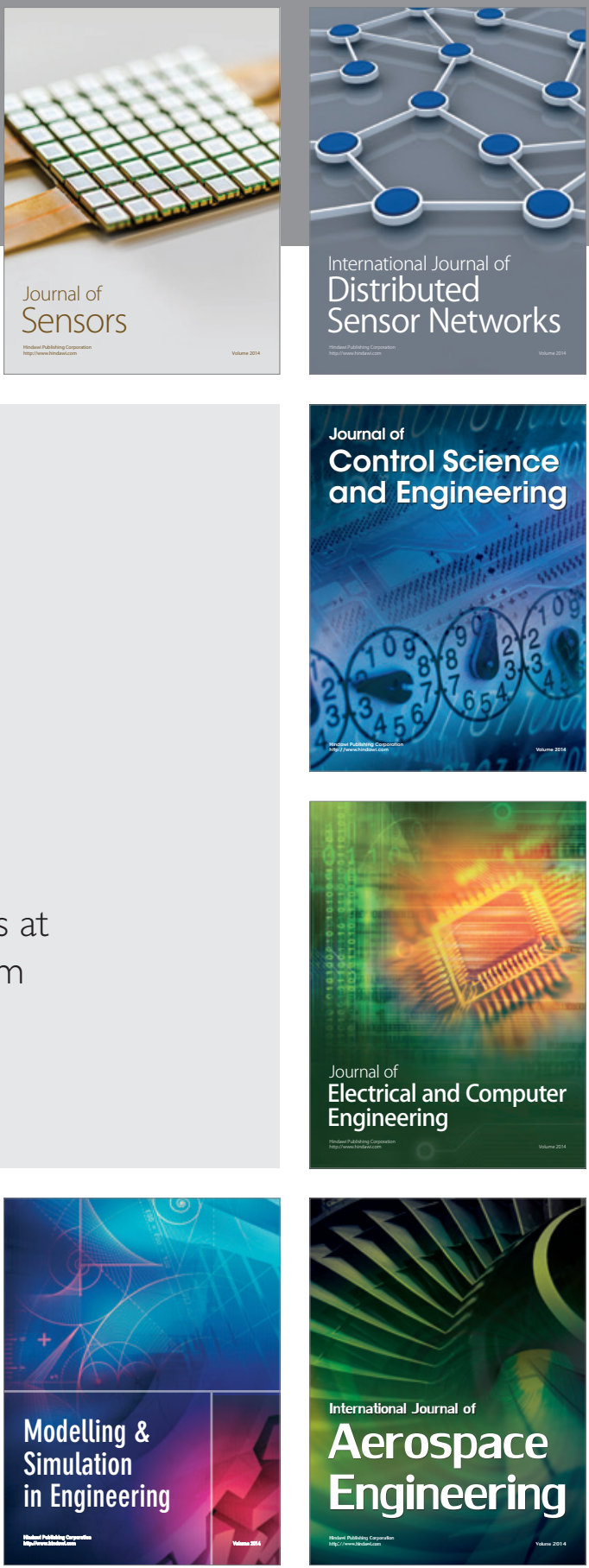

Journal of

Control Science

and Engineering
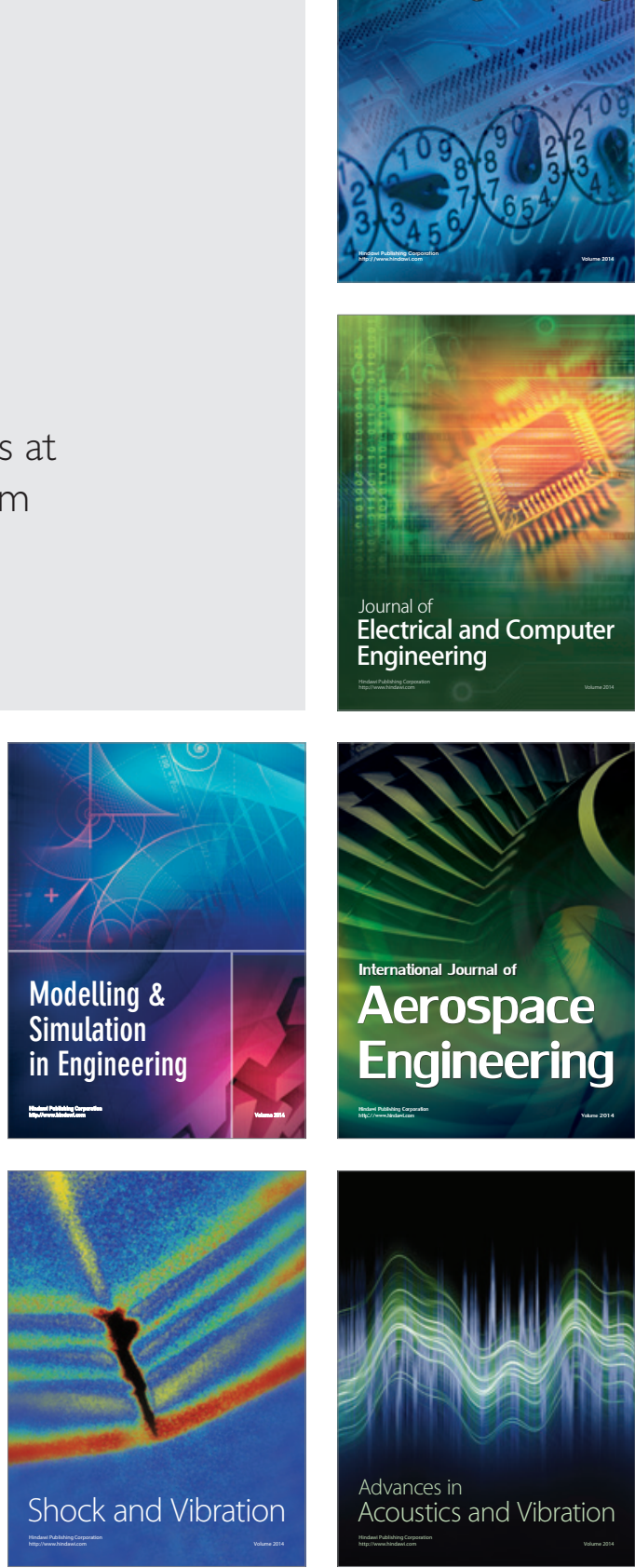